\title{
Kapitel 5 \\ Der Satz: wie wir organisieren, was wir zu sagen haben, und wie wir zeigen, was uns wichtig ist
}

\section{Einstieg}

„Nach Herbert Wehner, Dietrich Bonhoeffer und Erwin Rommel spielt Ulrich Tukur jetzt den legendären Tierschützer Bernhard Grzimek.“

So zitiert der „Hohlspiegel“ vom 4. April 2015 aus dem „Hamburger Abendblatt“. Wer spielt hier wen? Zwar wissen wir, dass Ulrich Tukur schon Wehner, Bonhoeffer und Rommel gespielt hat und sich ,jetzt“ den Grzimek vornimmt. Aber allein dem Wortlaut nach könnten auch Wehner, Bonhoeffer und Rommel schon vor Tukur in die Rolle von Bernhard Grzimek geschlüpft sein.

Wie kommt das? Offenbar können wir die Phrase mit der Präposition nach unterschiedlich beziehen, mal auf das Subjekt, den Ausdruck Ulrich Tukur. Dann verstehen wir ein Nacheinander der Darsteller. Mal beziehen wir die Phrase auf das direkte Objekt, den Ausdruck den legendären Tierschützer Bernhard Grzimek. Dann verstehen wir ein Nacheinander des Dargestellten.

Sätze sind also Gebilde, bei denen es wesentlich auf die Beziehungen zwischen den Teilen ankommt. Und zwar nicht zwischen irgendwelchen Teilen, sondern den „großen Stücken“, den so genannten ,Satzgliedern“, die sich bei einer ersten Zerlegung ergeben. Vor allem solche Satzglieder können wir im Deutschen häufig auch an eine andere Stelle im Satz verschieben, ohne dass Unsinn entsteht. Im Beispielfall etwa so: „Ulrich Tukur spielt nach Herbert Wehner, Dietrich Bonhoeffer und Erwin Rommel jetzt den legendären Tierschützer Bernhard Grzimek." Hier würde sich an der beschriebenen Mehrdeutigkeit nichts ändern. Vertauschen wir dagegen in Das ist nicht oft der Fall die beiden Wörtchen nicht und oft ändert sich die Bedeutung: Das ist oft nicht der Fall. Welche Satzglieder in einem Satz zugelassen sind, wird einerseits von der Wertigkeit, der ,Valenz', des Verbs gesteuert. Subjekt und Objekte müssen im Valenzrahmen des Prädikatsverbs ihrer semantischen wie morphosyntaktischen Art nach vorgesehen sein. Diesen Satzgliedtyp, die Komplemente, haben wir daher schon im Kapitel zum Verb angesprochen. Sätze bestehen aber andererseits nicht nur kurz und knapp aus Prädikat + Komplementen. Wir schweifen aus, fügen alle möglichen ,Supplemente، hinzu: Die nach-Phrase und jetzt sind solche Supplemente, die zum Satzkern in unserem Beispielsatz hinzukommen. Mit diesen Gliedern des Satzes beschäftigen wir uns in Unterkapitel 2. 
Neben der inneren Ordnung nach Satzgliedern unterschiedlicher Art haben Sätze aber eine sicht- oder hörbare lineare Ordnung. Und diese ist es im Übrigen auch, die uns hilft, die innere Ordnung überhaupt herzustellen oder zu erkennen. Das haben wir gerade schon angesprochen. Das Nacheinander im Satz, seine ,Topologie', hat verschiedene Dimensionen und Funktionen. Im Deutschen spielt die Position des finiten Verbs hier eine zentrale Rolle. Das Finitum kann ganz vorn stehen (Spielt Tukur Grzimek); es kann als zweites ,Stellungsglied' erscheinen (Tukur spielt Grzimek) oder es kann den Satz abschließen (wenn Tukur Grzimek spielt) oder vielmehr dessen zentrales Feld, das ,Mittelfeld‘, gegenüber einem ,Nachfeld‘ abgrenzen (wenn Tukur Grzimek spielt nächstes Jahr). Wir haben die Beweglichkeit des finiten Verbs schon in Kapitel 2, Abschnitt 5.2 kennen gelernt. Dort ging es um den Zusammenhang zwischen Verbstellung und ,Satzmodus', zwischen Sprachstruktur und Sprechakt. Aber die Verbposition strukturiert darüber hinaus den Satz in mindestens drei unterschiedliche Felder, die den dort verteilten Satzgliedern gegebenenfalls unterschiedliche Bezüge zuweisen können, vor allem aber jeweils unterschiedliche Rollen in der Informationsaufbereitung oder unterschiedliches Gewicht. Sagen wir etwa Den Grzimek spielt der Tukur statt dem üblicheren und „normaleren“ Der Tukur spielt den Grzimek, so wäre diese Formulierungsvariante angemessen, wenn zum Beispiel der Satz vorher gelautet hätte: Die Rollenbesetzung für den geplanten Film ist schon teilweise bekannt. In diesem Fall stünde das Thema ,Rollen' schon im Raum, und im ,Vorfeld‘ des Folgesatzes, also vor dem Verb, könnte gleich die zentrale Rolle, nämlich die des Grzimek, genannt werden. Die „Drei-Felder-Wirtschaft“ des Deutschen wird so im Übrigen von anderen europäischen Sprachen nicht geteilt. Mit seiner teilweise fixen, teilweise aber auch variablen Satztopologie steht das Deutsche z. B. zwischen Französisch und Englisch auf der einen und Polnisch auf der anderen Seite: In den ersteren gibt es weniger topologische Freiheiten, im Polnischen eher mehr. Das so vielseitige Thema der linearen Ordnung im Satz ist Gegenstand von Unterkapitel 3.

Was eigentlich ist ein Satz? Ich stelle die Diskussion dieser Frage ans Ende, nicht an den Anfang des Kapitels, erörtere sie also in Unterkapitel 4. Dabei vertraue ich darauf, dass sich meine Leserinnen und Leser ganz selbstverständlich auf die eine Lesart eingelassen haben, die ich ihnen zuvor präsentiert habe: der Satz als grammatische, als syntaktische Einheit, bestehend mindestens aus dem verbalen Prädikat und seinen Komplementen. Man kann den Terminus aber auch anders verstehen und dabei den Satz als kleinste Einheit der Rede oder des kommunikativen Handelns im Auge haben. Kann man die Sehweisen aufeinander beziehen oder gar miteinander versöhnen? 


\section{Die Glieder des Satzes: Wo, wann, warum spielt die Musik? Und wie finden wir das?}

\subsection{Die verschiedenen Arten der Supplemente}

Im schulischen Grammatikunterricht lernen wir, uns die innere Form des Satzes über Fragen zu erschließen. Mit wer oder was fragen wir nach dem Subjekt, mit wen oder was nach dem direkten, mit wem nach dem indirekten Objekt, etwa bei einem Satz wie „Wir schulden den Bürgern gute Daten” (Süddeutsche Zeitung vom 24.05.2003). Bei diesen Komplementen des verbalen Prädikats stimmt der Kasus des Fragepronomens mit dem Kasus der Phrase, die als Antwort geliefert wird, überein: Nominativ für das Subjekt, Akkusativ für das direkte Objekt, Dativ für das indirekte. Aber wir haben ja in der Regel noch zahlreiche andere Fragen zu dem vom Verb und seinen Komplementen knapp entworfenen Szenario. ${ }^{1}$ Wir stellen sie - getreu dem Motto der Sesamstraße - z. B. mit wieso, weshalb, warum oder auch oft mithilfe von wo, wann, wie usw. Diese Frageadverbien, nicht flektierbare $w$-Wörter, sollen uns zu Informationen über die „Umstände“ eines Szenarios führen, wie man so sagt. Die eingeforderten ,Umstandsangaben', ,adverbialen Bestimmungen“ oder auch kurz ,Adverbiale، - so nannte oder nennt man sie in der Schulgrammatik können als Supplemente im Satz bereits gegeben sein, z. B. wenn unser Beispielsatz gelautet hätte: Heute schulden wir in Europa den Bürgern aus demokratischer Verantwortung gute Daten in Form verständlicher Statistiken. Oder aber sie werden auf Nachfrage nachgeliefert.

Welche und wie viele solcher Supplemente gibt es? Diese Frage wird von den Grammatikern nicht klar beantwortet. Denn anders als bei den Komplementen, die ja durch die Verbvalenz präfiguriert und mehr oder weniger festgelegt sind, sind die Supplemente grammatisch frei - zumindest mehr oder weniger. So erfolgt auch ihre Klassifikation - anders als im Komplementbereich - nicht nach der grammatischen Form oder Funktion, sondern nach ihrem Beitrag zum dargestellten Szenario, seiner Inszenierung oder seiner kognitiven und evaluativen Verarbeitung. Da sind an vorderster Stelle Temporal- und Lokalangaben: Das Wann und Wo, die Verankerung in Zeit und Raum, gehört zu jedem singulären Ereignis als dem Prototyp eines dargestellten Szenarios hinzu. Angaben zur Zeitdauer, ,Durativangaben', wie stundenlang, seit heute Morgen ${ }^{2}$ setzen bereits ein Ereignis von einer merklichen Dauer voraus, sind also mit ,telischen', auf ein Ende fixierten oder ,punktuellen‘ Prädikationen wie den Gipfel erreichen oder das Portemonnaie stehlen / finden kaum vereinbar. Allenfalls in Kombination mit einer ,Frequenzangabe“ wie dreimal, mehrfach, zum x-ten Mal ergeben sie wieder Sinn, denn dann ist ja von mehreren Einzelereignissen die Rede, die sich insgesamt über einen gewis- 
sen Zeitraum erstrecken: „Der 22-jährige Arbeitslose soll seit Juli mehrfach Geldbörsen und Taschen gestohlen haben“ (Braunschweiger Zeitung vom 25.10.2005).

Auch ,Modalangaben“ (wie?), und ,Instrumentalangaben“ (womit?) oder ,Komitativangaben‘ (mit wem?, unter welchen Begleitumständen?) setzen bestimmte Ereignistypen voraus. Handlungen werden typischerweise mit Werkzeugen oder Instrumenten durchgeführt: Man schneidet mit einem Messer, Kinder rechnen zunächst mit den Fingern. Hier also sind ,Instrumentalangaben' erwartbar. Diejenigen Komitativangaben, die einen belebten Begleiter benennen - er kann natürlich auch durch Abwesenheit glänzen -, beziehen sich in der Regel auf Ereignisse oder Zustände mit einem menschlichen Träger wie in Der Mann geht mit seinem Hund spazieren, Sie ist diesmal ohne ihre Freundin in Frankfurt.

,Kausalangaben' beantworten die Frage nach dem Warum und Wieso, während ,Finalangaben“ benennen, wozu etwas geschah: „Er habe dann aus Angst, und um sich und seine Freundin zu schützen, geschossen“ (dpa vom 19.02.2013). Die Angst ist der Grund, das Motiv des Handelns, der Schutz der Freundin dessen vorgebliches - Ziel. Obwohl Gründe und Motive vor einem Geschehen zu liegen scheinen und Ziele oder Zwecke dessen beabsichtigte Folgen sind, liegt beiden eine Verknüpfung von Voraussetzung und Folge zugrunde. Man kann sie also auf eine Wenn-dann-Aussage, ein ,Konditionalgefüge‘, zurückführen. Im Fall unseres Belegs also z. B. „Wenn man Angst hat, schießt man unter Umständen“. Bei einer kausalen Verknüpfung kommt dann noch hinzu, dass die Voraussetzung tatsächlich vorliegt, in unserem Fall der Schießwillige seiner Aussage nach wirklich Angst hatte. Für die Finalangabe gilt entsprechend: „Wenn man jemanden schützen will, muss man unter Umständen schießen.“ Auch wenn es sich hier um abwegige Überlegungen handeln mag, so zeigt sich doch die grundlegende Bedeutung der Schemata rationalen Argumentierens für einen ganzen Komplex von Umstandsangaben: Nicht nur Kausal- und Finalangaben, sondern auch ,Konsekutivangaben“ und sogar ,Konzessivangaben“ gehören dazu. Konsekutivangaben sind sozusagen die Umkehrung von Kausalangaben: Wenn der Betreffende aus Angst geschossen hat, dann hatte er offenbar Angst und schoss infolgedessen. ,Konzessivangaben‘ hingegen nennen Gründe, die eigentlich gegen etwas sprechen, aber nicht zum Zuge kommen oder kommen können. Unser Beleg hätte ja auch lauten können: „Er habe dann trotz seiner Angst geschossen.“ Oder: „Er habe dann, obwohl er solche Angst hatte, geschossen.“

Gerade bei den konditional fundierten Supplement-Typen kann man feststellen, dass Sprecher ihre Aussagen nicht nur auf der Ebene der thematisierten Sachverhalte zum Beispiel kausal oder konzessiv ergänzen, sondern mit (fast) denselben sprachlichen Mitteln auch auf die ,epistemische‘, also wissensbezogene, oder gar die illokutive Ebene umschwenken können. Auf das „fast“, das z. B. Linearität und Prosodie betreffen kann, kommen wir im nächsten Abschnitt 
zurück. Während auf der Sachverhaltsebene ein weil-Satz die Ursache einer Wirkung beschreibt, kann ein epistemischer weil-Satz die Begründung formulieren, die jemand sich für etwas zurechtgelegt hat. Wenn der kleine Christian auf die Frage, wie denn das Christkind auf die Erde herunterkomme, antwortet: „Vielleicht fliegt's ja, weil, das ist ja immer so leise“ (Mannheimer Morgen vom 18.12.1995), dann hat er eine solche für ihn plausible Begründung geäußert. Er schließt aus der lautlosen Annäherung zurück auf einen möglichen Flugmodus. Nicht nach dem Schema: „Wenn man leise ist, dann fliegt man“, sondern umgekehrt: „Wenn man fliegt wie ein Vogel, dann macht man nicht viel Lärm“. Also kann es sein, dass die lautlose Annäherung des Christkinds auf das Fliegen zurückzuführen ist. Wenn andererseits Dirk Nowitzki im Stern-Interview vom 14. August 2008 sich so äußert: „Während der Saison schreiben wir uns ab und zu eine E-Mail, obwohl, ich muss zugeben: Ein großer Poet bin ich nicht“, dann ordnet er seine Aussage als Zugeständnis, als Einräumung ein, bewegt sich also auf der illokutiven Ebene.

Die Beispiele haben gezeigt: Umstandsangaben können nicht nur durch Adverbien wie ein temporales dann, ein lokales hier, ein konsekutives infolgedessen, ein konzessives trotzdem und Präpositionalphrasen wie nach zwei Stunden, in Frankfurt, trotz seiner Angst realisiert werden, sondern auch durch Nebensätze. Temporale Nebensätze werden z. B. durch als, wenn, nachdem oder während eingeleitet, kausale typischerweise durch weil und konzessive durch obwohl. Dabei werden zwei Szenarios ins Verhältnis gesetzt und durch die einleitenden ,Subjunktoren` - man nannte diese Wörtchen traditionell ,unterordnende Konjunktionen‘ - wird das Verhältnis zwischen dem Szenario des Hauptsatzes und dem Szenario des Nebensatzes relativ klar benannt. Finalangaben werden oft durch damit-Sätze ausgedrückt, daneben aber auch durch Infinitivkonstruktionen mit um ... zu, wie wir in dem Beleg oben gesehen haben, wo einer schoss, um sich und seine Freundin zu schützen.

Man kann noch weitere Typen von Supplementen unterscheiden, denkt man nur an das ,adversative“ während wie in Doch während die einen feierten, hatten andere Grund zum Ärgern oder das ,substitutive ' anstatt wie in Anstatt zu feiern, hatten wir Grund zum Ärgern. Diese Typen wie schon den konzessiven Typ kann man nicht mit einem entsprechenden Frageadverb erfragen: Wann / Wo / Wie / Warum ist das geschehen? Das sind gute Fragen, aber nicht: ${ }^{*}$ Trotzwessen / Währendwessen / *Anstattwessen ist das geschehen? Ebenfalls nicht erfragbar ist der gesamte Komplex von Angaben, die man als ,Kommentarglieder‘ bezeichnet hat. Bei ihnen geht es nicht um die Situierung oder Relationierung von Szenarios als solchen, sondern um Zugaben aus der Sicht des Sprechers. Auch dies ist ein weites Feld: Sprecher können z. B. die Gültigkeit ihrer Aussagen bekräftigen mit tatsächlich, ohne Zweifel oder sie auch relativieren mit vielleicht, wahrscheinlich. Sie können sie bedauernd oder erfreut kommentieren, etwa mit leider und gottsei- 
dank. Sie können mit wie erhofft ihre Stellung zu Sachverhalten, von deren Eintreten sie (noch) nichts wissen, bekunden oder mit erwartungsgemäß oder zu meinem größten Bedauern ihren Senf zu bereits Eingetretenem geben.

Schließlich und endlich werden durch Wörtchen wie eben, halt, ja oder doch Einschätzungen des Sprechers ausgedrückt, deren „Sinn“ nur schwer erfasst, geschweige denn umschrieben werden kann. „So funktioniert eben der Mensch” heißt es in einem Beitrag der Süddeutschen Zeitung vom 4. April 2009. Durch eben scheint der Autor oder die Autorin darauf abzuheben, dass der Sachverhalt, um den es geht, eine allseits bekannte und daher nicht mehr in Frage stehende Tatsache ist. Würde sich an dieser Note viel ändern, wenn stattdessen das eher süddeutsche halt stände? Wohl kaum. Der Einsatz von ja würde vielleicht die Aussage eher als Antwort auf eine unausgesprochene Frage qualifizieren und gleichzeitig ebenfalls andeuten, dass es dabei um eine bekannte Tatsache geht. Wäre dagegen die Aussage mit doch „gewürzt“ worden, so würden wir im Hinterkopf des Autors vielleicht einen antizipierten Widerspruch vermuten, dem er gleich zuvorzukommen und damit seine Aussage noch zu bekräftigen versucht. Ginge es um eine Frage, dann würden die Partikeln denn oder blo $\beta$ gut passen, und man könnte sie sogar noch anhäufen: „Funktioniert der Mensch denn so?“ „Warum funktioniert der Mensch (denn) bloß so?“

Wie man sieht, steht der Erklärungsaufwand, den man hier betreiben muss, in keinem Verhältnis zur Kürze und subtilen Wirkung dieser so genannten ,Abtönungspartikeln“. Nicht von ungefähr waren diese auch „Würzwörter“ genannten Ausdrücke vor allem in den 1980er und 1990er Jahren besonders beliebte und intensiv beforschte Untersuchungsgegenstände der germanistischen Linguistik. „Sie tragen zur Einpassung der kommunikativen Minimaleinheit in den jeweiligen Handlungszusammenhang bei, indem sie auf den Erwartungen und Einstellungen des Sprechers und der Adressaten operieren“, so und ähnlich wird ihre Funktion zu fassen gesucht. ${ }^{3}$ Besonders betont wurde, dass es sich dabei um ein Spezifikum des Deutschen zu handeln scheint: Vor allem in dieser Sprache wurden Adjektive (wie eben, bloß), aber auch die Antwortpartikel ja oder ,Konnektoren', also Satzverknüpfer wie denn oder doch so weit grammatikalisiert, dass sie nur noch auf einer quasi nicht-verbalen Ebene funktionieren. ${ }^{4}$ So werden z. B. Englisch, Französisch oder Polnisch im Vergleich zum Deutschen als „partikelarme“ Sprachen bezeichnet. Nur dem Ungarischen wird ein ähnlicher Reichtum an solchen Wörtchen bescheinigt. ${ }^{5}$ Die deutschen Abtönungspartikeln sind aber eben nicht nur durch funktionale, sondern auch durch formale Gemeinsamkeiten ausgezeichnet. Sie können z. B. nur im Mittelfeld (vgl. Abschnitt 3.4) und dort zudem gehäuft auftreten. Eine solche Profilierung als formale Klasse findet man auch im Englischen nicht, selbst wenn sich z. B. Parallelen zwischen deutsch denn und englisch then oder deutsch schon und englisch already zu erge- 
ben scheinen. Ein Beispiel: Eine deutsche Frage wie „Was wirst du denn (bloß) tun?“ kann im Englischen durch „What are you going to do then?“ übersetzt werden. Das könnte aber auch interpretiert werden als ,Was wirst du dann tun?`6

Aus sprachvergleichender Perspektive sind daneben auch die Muster interessant, nach denen die zahlreichen Präpositionen, Adverbien und Subjunktoren gebildet werden, mit denen man temporale, kausale und andere Verhältnisse ausdrückt. Ein wichtiges sprachübergreifendes Prinzip scheint dabei zu sein, dass die Verhältnisse im Raum als Übertragungsquelle zunächst für zeitliche Relationen dienen, dann auch für die abstrakteren konditional fundierten und weitere Beziehungen. Deutsch an, bei, in, nach und vor beispielsweise haben neben einer lokalen auch eine temporale Interpretation: an der Straße versus an einem Montag, bei Frankfurt versus bei Sonnenuntergang, in der Schule versus in einer Stunde, nach Frankfurt versus nach Sonnenuntergang, vor dem Haus versus vor einer Stunde. Auch in anderen Sprachen sind lokale Präpositionen gleichzeitig temporal. Aber die Zuordnungen sind nur teilweise parallel. Heißt es im Deutschen: bei Tag und Nacht, am Tag und in der Nacht, sieht es im Englischen so aus: by night and day, in the day and in the night und im Polnischen $w$ dzien $i$ $w$ nocy. Im Polnischen wird also die lokale Standardpräposition in der Bedeutung ,in' gebraucht. Im Französischen wird die zeitliche Lokalisierung oft ohne Präposition ausgedrückt, nicht nur bei formelhaften Verbindungen wie jour et nuit, das wir auch im Deutschen kennen (vgl. Er arbeitet Tag und Nacht), sondern generell, z. B. Il travaillait le lendemain ,Er arbeitete am Tag danach‘. Im Deutschen kennen wir eher bei Zeitdauerangaben einen ,Akkusativ der Erstreckung' wie in Er arbeitete den ganzen Nachmittag.

Kausale Präpositionen sind ebenfalls sprachübergreifend häufig gleichlautend mit lokalen. Im Deutschen gilt dies in erster Linie für emotionale Beweggründe: vor Angst weinen, aus Eifersucht durchdrehen, aber auch aus diesem Grund, aus Versehen. Auch das Polnische scheint mit der Präposition $z$ oder $z e$,aus ${ }^{`}$ wie in $z e$ strachu ,aus Angst', $z$ głodu ,aus/vor Hunger‘ die Vorstellung zu bedienen, unser Verhalten werde durch von innen nach außen drängende Kräfte bewirkt. Im Ungarischen übernimmt häufig ganz parallel dazu das Suffix -böl/-ból für den Kasus ,Elativ“, also den Kasus des „woher?“, die Markierung von Gründen wie in barátságból ,aus Freundschaft‘. Aber Vorsicht: Es handelt sich um längst „tote“ Metaphern, über die der Sprachgebrauch souverän hinweggeht. Vielleicht geht es ja auch bei der Entstehung der Praxis ,Kausales durch Lokales‘ eher um den Gesichtspunkt der Sprachökonomie - durch Recyceln einer lokalen Präposition für kausale Zwecke spart man sich die Erfindung neuer Ausdrucksmittel - als um psychologische Annahmen einer längst vergangenen Zeit.

Im Englischen ist in erster Linie for zu nennen for fear ,aus Angst‘, for some reason ,aus irgendeinem Grund‘, aber auch just for fun ,nur zum Spaß` oder 
auch, etwas weniger beliebt, ,nur aus Spaß‘. ${ }^{7}$ Hier kann offenbar die Perspektive zwischen der Nennung des Grundes (aus) und des Zwecks ( $z u$, englisch for) wechseln. Gründe erscheinen uns oft als Zwecke. Denken wir bei der Angabe des Grundes eher egoistisch an die Gefühle, die aus uns aufsteigen, und bei der Nennung von Zwecken altruistischer an das, was für uns und andere daraus folgen soll? Eher spielt wohl die alte etymologische Verwandtschaft zwischen vor und für bzw. germanisch fora und furi eine Rolle. Beide Varianten gehen auf eine erschlossene proto-indoeuropäische Wurzel * per zurück. Diese wiederum ist in den verschiedensten Tochtersprachzweigen nachweisbar. Das Französische kleidet übrigens ähnlich wie das Englische Gründe gern in Zwecke wie bei pour cette raison ,aus diesem Grund' oder aber bedient sich der ebenfalls auch sonst verbreiteten Metaphorik des Wegs (vgl. deutsch wegen), Mittels oder Instruments. Hier kommt dann eine weitere Variante von *per zum Zug, wie in par hasard ,aus Versehen' oder par courtoisie ,aus Höflichkeit‘. Der externe Auslöser einer Gemütsbewegung oder Gefühlsäußerung, der Stimulus, wird oft mittels der Präposition über gekennzeichnet: Wir lachen über einen Witz; aber wir lachen aus Übermut oder vor Freude. Im ersteren Fall stehen wir dem Anlass der Emotion als Beobachter gegenüber, im zweiten Fall sind wir die unmittelbar Betroffenen, die sich gegen Gefühle kaum wehren können. Es sei auch noch angemerkt, dass die lokale Vorstellung des Sich-in-etwas-Befindens ihrerseits genutzt wird, um emotionale Befindlichkeiten - ohne eine notwendige kausale Note - zu beschreiben. Gefühle umschließen uns dann sozusagen wie Gefäße. Man vgl. in Wut/Rage sein / handeln oder englisch in rage, französisch en colère. Auch auf Polnisch ist die Vorstellung lebendig, wenn die Präposition $w$ oder we zum Zuge kommt, wie in w złości ,im Zorn'. Im Ungarischen wird das Suffix -ben/-ban für den Kasus ,Inessiv“, also den Kasus des „wo?“ gebraucht, wie in méregben oder dühben, in Wut'.

Bei kausalen Nebensätzen wiederum ist häufig das Prinzip des post hoc ergo propter hoc, des ,danach also deshalb،, wirksam: Zeitlich Vorangehendes wird als Verursachendes gedeutet. ${ }^{8}$ Ähnlich wie beim Vorgehen des oben erwähnten kleinen Christian wird hier von einem vorliegenden Sachverhalt auf einen verursachenden rückgeschlossen. Dabei ist natürlich das rein zeitliche Vorher nicht hinreichend für eine Kausalbeziehung. Logisch gültig ist das Prinzip nicht. Aber immerhin möglich ... Im Deutschen können z. B. nachdem-Sätze gegebenenfalls kausal interpretiert werden. Man vergleiche folgenden Beleg aus einem Sitzungsprotokoll des baden-württembergischen Landtags vom 25. März 1999: „Sie wissen auch, dass es, nachdem Rot-Grün in Bonn regiert, nicht so leicht ist, Geld für sinnvolle Dinge aufzutreiben, sondern dass man Prioritäten zu setzen hat.“ Es kann durchaus gemeint sein „weil Rot-Grün in Bonn regiert“; beweisen lässt sich das allerdings nicht. Auch seit konnte vergleichbar englisch since bis zum Mittel- 
hochdeutschen kausal interpretiert werden. Heute wird nur noch der seltene und altertümelnde Subjunktor sintemal(en) kausal gebraucht. „Die Rechnung dafür wird jetzt mit Verzugszinsen fällig, sintemal auch bei Kirchens Personal- und Baukosten die großen Happen in den Haushalten sind“ (ZEIT vom 15.09.1995).

Kommen wir noch auf einen Supplementtyp zu sprechen, der nicht so recht unter die ,Umstandsangaben“ passt. Wenn man gut gelaunt das Büro verlässt oder voller Wut die Türen zuknallen lässt, dann geht es eigentlich nicht um das Wie der betreffenden Handlung, sondern um die Befindlichkeit des Akteurs oder der Akteurin während des Handlungsvollzugs. Man spricht also eine zusätzliche Prädikation über diese Person aus. Diesen Typ bezeichnet man daher als ,freies Prädikativ‘. Charakterisiert wird in erster Linie das vom Subjekt Bezeichnete, aber es kann auch um die Befindlichkeiten des im Objekt Genannten gehen: „Und Weißensee hat an diesem Spätsommertag offenbar keine Kneipe, die nicht Betriebsferien macht und den Besucher hungrig stehen läßt“ (ZEIT vom 23.09.1966). Hungrig sind hier die Besucher. Neben freien Prädikativen, die den jeweils aktuellen Zustand beschreiben, gibt es auch solche, die den Zustand beschreiben, der für den Betroffenen aus dem vom Verb bezeichneten Ereignis resultiert. Einer kann sich selbst grün und blau ärgern, eine mag sich die Fußnägel grün oder blau anmalen. Oftmals sind resultative Prädikative gar nicht freie Zugaben, sondern setzen eine ohne sie gar nicht gegebene Konstellation von Verb-Komplementen voraus; sie erzeugen sozusagen einen veränderten Valenzrahmen, in dem sie selbst als Komplement integriert sind: Man kann eine(n) andere(n) müde oder an die Wand quasseln, aber nicht jemanden quasseln. Bei machen (Das macht mich glücklich) oder zu etwas bringen (Das bringt sie zur Weißglut) ist die Herbeiführung eines Resultatzustandes Teil der normalen Verbbedeutung, und das Prädikativ gehört ebenfalls eindeutig in den Valenzrahmen.

Die sprachliche Umsetzung freier (oder auch valenzgebundener) Prädikative divergiert recht erheblich zwischen europäischen Sprachen: Im Französischen und Polnischen beispielsweise werden prädikative Adjektive - anders als im Deutschen oder Englischen - flektiert und können mit der Bezugsphrase kongruieren. Das gilt nicht nur in Kopulasätzen, man vgl. französisch Il est fatigué / Elle est fatiguée, polnisch Jest zmęczony / Jest zmęczona ,Er / Sie ist müde‘, sondern auch, wenn sie als freie Prädikative gebraucht werden. Durch Kongruenz kann so ggf. der Bezug - ob Subjekt oder Objekt - verdeutlicht werden: Zwar würde bei Peter isst das Fleisch roh auch im Deutschen kaum ein Zweifel entstehen, in der französischen Version Pierre mange la viande crue zeigt die feminine Form crue eindeutig Objektbezug an. Im Ungarischen wird ein Adjektiv als freies Prädikativ formal nicht von der Modalangabe unterschieden. Während aber im Deutschen - das ja hier auch nicht formal differenziert - die unflektierte Adjektivform gesetzt wird, erscheint im Ungarischen das vom Adjektiv abgeleitete 
Adverb. Das Fleisch oder den Fisch roh zu essen, erscheint dann - im Ungarischen explizit, im Deutschen eher durch die adjektivische Form verdeckt - sozusagen als eine rohe Weise, Fleisch oder Fisch zu essen, was ja als gar nicht so abwegig erscheinen mag.

Was Resultativkonstruktionen angeht, sind wohl am ehesten im Deutschen Adjektive als Bezeichnungen für den Nachzustand üblich. Bei uns gibt es sogar die Tendenz, resultatives Objektsprädikativ und Verb zusammenwachsen zu lassen und sogar übertragen zu verwenden: etwas glatt bügeln, totreiten, schönreden oder schwarzmalen, sich krummlegen, ein Unternehmen, (z. B. die Deutsche Bahn) kaputtsparen. ${ }^{9}$ Meist wird in anderen Sprachen mit Umschreibungen nach dem Motto ,durch X-en in den Zustand Y versetzen` gearbeitet. ${ }^{10}$

\subsection{Mut zur Mehrdeutigkeit}

Mehrfach sind wir bereits darauf gestoßen, dass Supplemente verschiedene Bezüge auf andere Teile des Satzes - bzw. das jeweils von diesen Bezeichnete haben können. Manchmal ist diese Mehrdeutigkeit auch durch Umstellungen nicht wegzukriegen (man vergleiche unser Einstiegsbeispiel). Dann liegt es nicht an mehreren unterschiedlichen Satzstrukturen, die nur auf der Oberfläche zusammenfallen, sondern, wenn man so will, an einer Unzulänglichkeit der sprachlichen Möglichkeiten gegenüber einer komplexeren Realität. Sprachlich behandeln wir Nach Wehner spielt Tukur Grzimek genauso wie Nach 2017 spielt Tukur Grzimek, obwohl letzteres nur eine, ersteres aber zwei Deutungsmöglichkeiten hat. In den meisten Fällen von Mehrdeutigkeiten aber gilt: Die eindimensionale lineare Folge entspricht zwei oder noch mehr ,inneren Ordnungen“. Die innere Ordnung im Satz, die man selbstverständlich immer schon gesehen hat, wird seit dem Strukturalismus explizit als eine hierarchische Ordnung begriffen. Man visualisiert die Abstufung in der Aufteilung des Satzes in der Regel durch Baumstrukturen: Zunächst wird der Satz in seine Hauptäste, die ,unmittelbaren Konstituenten“ zerlegt, diese wiederum in ihre Konstituenten - ,mittelbare Konstituenten“ des Satzes -, bis hinunter zu einzelnen Wortformen, oder gar bis zu den Morphemen als Wortbausteinen. So ist zumindest das klassische Vorgehen, bei dem man nur zerlegt, was auf dem Papier steht oder zu Ohren kommt, also darauf verzichtet, in den Strukturen nicht real existierende Elemente, für die man Evidenzen zu haben glaubt, erscheinen zu lassen. ${ }^{11}$

Ich zeige jetzt an einem Beispiel aus dem „Hohlspiegel“ (Spiegel vom 21.12.2013) exemplarisch, wie sich hinter einer linearen Struktur zwei verschiedene innere Strukturen verbergen können, in Form von zwei verschiedenen Baumstrukturen. 
Meine Bäume stehen auf dem Kopf: Das entspricht der analytischen Vorgehensweise beim Sprachverstehen: Wir tasten uns verstehend von links nach rechts bzw. vom Früheren zum Späteren vor und synthetisieren in rasender Eile Wörter zu Satzgliedern und Satzglieder zum Satzganzen. ${ }^{12}$ Ein Baum, bei dem wie üblich der Gipfel nach oben und die Wurzeln nach unten zeigen, entspräche eher der Produktionsperspektive. Man muss ihn dazu nur horizontal spiegeln.

Das Beispiel entstammt einer Schlagzeile, ist also durch Weglassung der Artikel besonders kurz und knapp gestaltet. Aber das ändert nichts an der strukturellen Mehrdeutigkeit, die uns hier interessiert. Und natürlich verdankt das Ganze seine Hohlspiegel-Würdigkeit der Tatsache, dass wir zwar zunächst in die Irre geführt, durch die Absurdität der einen Interpretation aber rasch eines Besseren belehrt werden: „Polizist erschießt Mann mit Machete“.

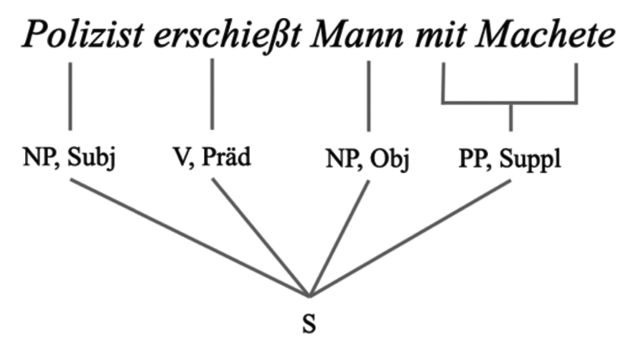

Abb. 12: Struktur a.

\section{Polizist erschießt Mann mit Machete}

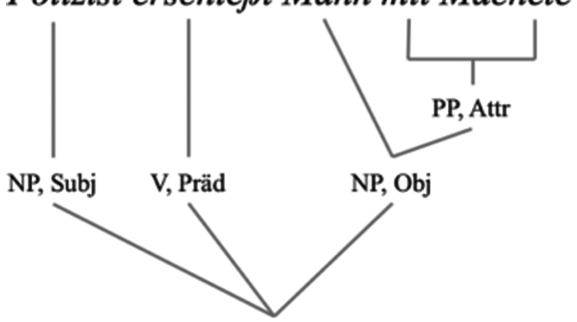

Abb. 13: Struktur b.

Bei Struktur a), der absurden, ist mit Machete unmittelbare Konstituente des Satzes, oder in relationaler Ausdrucksweise ein Supplement, ein echtes „Satzglied“. Bei Struktur b) hingegen, der gemeinten, ist mit Machete nur mittelbare Konstituente, als Attribut Teil des direkten Objekts.

Vieles aus der Hohlspiegel-Blütenlese ist von ähnlicher Art. Ein Teil des Satzes kann als Supplement auf Satzebene oder als Attribut interpretiert werden. 
Und eine der beiden Lesarten bringt uns zum Schmunzeln. Genau gleich gestrickt wie unser Beispiel eben ist diese Schlagzeile: „Polizist erschießt Mann mit Messer“ (Hohlspiegel, Spiegel vom 06.05.2017). Oder auch: „Polizist schießt mit Messer bewaffneten Mann nieder“ (Hohlspiegel, Spiegel vom 23.09.2017). Hier gehört mit Messer zu bewaffneten, wieder nicht als Umstandsangabe zur polizeilichen Schießaktion. Oder, wie es in einem Kluftinger-Krimi heißt: „Es ist jedenfalls so, dass viele Morde von Hausärzten unentdeckt bleiben. “13

Ich nenne einige weitere Beispiele dieser Art. Im Anschluss kennzeichne ich die alternativen inneren Strukturen mithilfe von eckigen Klammern: Sie zeigen die Grenzen von Konstituenten auf und sind damit ein probates Mittel, um Baumstrukturen in angereicherte lineare Strukturen umzusetzen. Das erste Beispiel zeigt zudem, dass auch Infinitivkonstruktionen im Prinzip wie Sätze gebaut sind; es fehlt das Subjekt, und das Verb steht natürlich im Infinitiv.

Kapitän Philipp Lahm war es dann um 22.54 Uhr vorbehalten, den riesigen Pokal mit den Ohren hochzustemmen.

(Hohlspiegel, Spiegel vom 03.06.2013)

[den riesigen Pokal] [mit den Ohren] hochzustemmen versus

[den riesigen Pokal mit den Ohren] hochzustemmen

Viele von ihnen mussten Zeltstädte auf der Insel Bohol verlassen, in denen sie seit dem Erdbeben im Oktober mit mehr als 200 Todesopfern lebten.

(Hohlspiegel, Spiegel vom 25.11.2013)

[in denen] [sie] [seit dem Erdbeben im Oktober] [mit mehr als 200 Todesopfern] lebten versus

[in denen] [sie] [seit dem Erdbeben im Oktober mit mehr als 200 Todesopfern] lebten

Eine Aktivistin der Frauengruppe Femen ist während der Weihnachtsmesse im Kölner Dom mit Kardinal Meisner halb nackt auf den Altar gesprungen.

(Hohlspiegel, Spiegel vom 06.01.2014)

[Eine Aktivistin der Frauengruppe Femen] [ist] [während der Weihnachtsmesse im Kölner Dom] [mit Kardinal Meisner] [halb nackt] [auf den Altar] [gesprungen] versus

[Eine Aktivistin der Frauengruppe Femen] [ist] [während der Weihnachtsmesse im Kölner Dom mit Kardinal Meisner] [halb nackt] [auf den Altar] [gesprungen]

Die an erster Stelle genannte Alternative ist jeweils diejenige, in der eine Präpositionalphrase als Supplement verstanden wird. Sie ist eigentlich, denke ich, schon nach dem Prinzip ,Strukturiere so einfach wie möglich' die erste Wahl aber jeweils ein Fehlgriff. In allen drei Fällen sollen wir die Präpositionalphrase als Attribut zum Kopf der jeweils vorangehenden Nominalphrase verstehen. Es 
geht also um den Pokal mit den Ohren, das Erdbeben mit mehr als 200 Todesopfern und die Weihnachtsmesse mit Kardinal Meisner.

Allerdings ist eine Fehlinterpretation unter umgekehrten Vorzeichen nicht ausgeschlossen. In der Überschrift „Befragung zu Alkoholverbot am Telefon?“ (Hohlspiegel, Spiegel vom 13.02.2016) - kurz etwa für „Darf eine Befragung zu Alkohol am Telefon durchgeführt werden?“ - ist am Telefon als Supplement zu verstehen. Wir tendieren aber zum Verständnis als Attribut, vielleicht weil wir die Fügung Alkohol am Steuer im Ohr haben.

Sprachliche Verständigung bedeutet also immer ein Umgehen mit potenziellen Mehrdeutigkeiten. Sie könnte uns daher durchaus als eine Schule der „Ambiguitätstoleranz“ dienen. ${ }^{14}$

\section{Lineare Ordnung}

\subsection{Kurzer Rückblick auf die lineare Ordnung als Indikator der Illokution}

Der Aussagesatz hat im Regelfall Verbzweitstellung - erinnern wir uns an Das Portemonnaie ist weg. In Ja-/Nein-Fragen wie Hast du wieder das Portemonnaie verlegt? und Aufforderungen wie Verlege bloß nicht wieder das Portemonnaie! haben wir es mit Verberststellung zu tun. Die Verbletztstellung wie in Dass du mir ja nicht das Portemonnaie verlegst ist typisch für Sätze, die mit einem Wörtchen wie dass oder wenn beginnen. Solche Sätze sind normalerweise ,Nebensätze‘, wie etwa in Ich weiß, dass du das Portemonnaie nicht verlegst. Nur ausnahmsweise formulieren wir mit ihnen selbstständige Ausrufe oder Wünsche. Die beiden Verbstellungstypen Verbzweit- und Verberstsatz - metaphorisch nannte man sie früher gern auch ,Kernsatz' und ,Stirnsatz - sind also klar verknüpft mit der Selbstständigkeit des betreffenden Satzes. Sie markieren ihn als eigene kommunikative Einheit. Dabei machen sie Vorgaben für den Satzmodus und durch diesen vermittelt für die möglichen Sprechakte, wenn auch keineswegs eindeutig.

Allerdings gibt es im Deutschen - keine Sprachregel ohne Ausnahme - auch Nebensätze mit Verbzweitstellung anstelle von Verbletztstellung, wie etwa in Ich hoffe, das geht klar anstelle von Ich hoffe, dass das klar geht. Das findet sich nicht nur wie im Beispiel oben bei Komplementsätzen, sondern auch bei Kausalsätzen mit weil oder auch bei obwohl-Sätzen. Wir hatten im vorangehenden Abschnitt bereits mit Christians Erklärung für die Reise des Christkinds zur Erde ein weil und mit der Bemerkung von Dirk Nowitzki ein Beispiel für ein obwohl, auf das jeweils ein Verbzweitsatz folgt. Häufig wird dies damit begründet, dass bei dieser „Hauptsatzstellung“ im Nebensatz das Hauptgewicht der Information eben auf dem Nebensatz liege, auf der Begründung, dem Einwand. Damit verkehre sich 
das informationelle Gewicht zwischen Haupt- und Nebensatz, und die Topologie trage dem Rechnung, indem sie dem Nebensatz Hauptsatzstellung zubillige.

Der Verbstellungstyp hat aber darüber hinaus weitere Funktionen. Zentral ist dabei die Portionierung des Satzes in unterschiedliche ,Felder‘.

\subsection{Die Felder des Satzes und wie sie bestellt werden}

Den Satz Die Ehefrau hat tatsächlich schnell das Portemonnaie gefunden, das ein Geschenk ihres Mannes ist teilen wir so in Felder ein:

Tab. 5: die Felder des Satzes.

\begin{tabular}{lllll}
\hline Vorfeld & $\begin{array}{l}\text { linker } \\
\text { Klammerteil }\end{array}$ & Mittelfeld & $\begin{array}{l}\text { rechter } \\
\text { Klammerteil }\end{array}$ & Nachfeld \\
\hline (1) Die Ehefrau & hat & $\begin{array}{l}\text { tatsächlich schnell } \\
\text { das Portemonnaie }\end{array}$ & gefunden & $\begin{array}{l}\text { das ein Geschenk } \\
\text { ihres Mannes ist }\end{array}$ \\
\hline
\end{tabular}

Der springende Punkt ist dabei die so genannte ,Satzklammer‘, auch ,Verbklammer' genannt. Typischerweise besteht sie wie in unserem Fall aus zwei Teilen: Den linken Klammerteil bildet hier die finite Form hat des Hilfsverbs, den rechten Klammerteil bildet die infinite Verbform gefunden des Vollverbs. Diese beiden Formen „umklammern“ das Mittelfeld: Dort befinden sich gleich drei Satzglieder: tatsächlich, schnell und das Portemonnaie. Allerdings ist mit das Portemonnaie das direkte Objekt, wie wir sehen werden, noch nicht abgeschlossen. Vor dem linken Klammerteil steht das Satzglied die Ehefrau als Besetzung des Vorfeldes. Nach dem rechten Klammerteil erstreckt sich das Nachfeld, hier besetzt durch den Relativsatz.

Wenn unser Satz - siehe (2) in Tab. 6 (s. folgende Seite) - z. B. im Präsens stände und das Prädikat nur aus der finiten Vollverbform findet bestände, ${ }^{15}$ hätten wir es nur mit einem linken Klammerteil zu tun. Das finite Verb ist nämlich im Verbzweitsatz, wie es dem Namen entspricht, auf diese Position abonniert. Mit dem Wegfall der rechten Klammer rutscht der Relativsatz ins Mittelfeld und steht damit dort, wo er eigentlich hingehört, nämlich unmittelbar nach dem Bezugswort Portemonnaie. Der Relativsatz ist ja Attribut dazu und damit auch Teil des direkten Objekts. Zuvor war er „ausgeklammert“ worden ins Nachfeld.

Auch im Verberstsatz - siehe (3) in der Tabelle - hält das Finitum seine Position in der linken Klammer. Nur das Vorfeld entfällt. Bei der Frage Hat die Ehefrau tatsächlich schnell das Portemonnaie gefunden? z. B. muss nun die Ehefrau 
automatisch ins Mittelfeld zurücktreten. Das Mittelfeld ist also nicht nur rein linear zentral, sondern auch als Ort, der praktisch alle Satzglieder (außer dem Prädikat) aufnehmen kann.

Im Verbletztsatz schließlich - siehe (4) in Tab. 6 - überlässt das finite Verb seinen Platz in der linken Klammer dem Nebensatzeinleiter, also einem Subjunktor wie dass, weil, wenn usw. oder auch einem Relativpronomen und wandert in die rechte Satzklammer oder gesellt sich zu dem oder den infiniten Teilen des Verbalkomplexes, also des Prädikats:

Tab. 6: die Felder im Verberst-, Verbzweit und Verbletztsatz.

\begin{tabular}{|c|c|c|c|c|}
\hline Vorfeld & $\begin{array}{l}\text { linker } \\
\text { Klammerteil }\end{array}$ & Mittelfeld & $\begin{array}{l}\text { rechter } \\
\text { Klammerteil }\end{array}$ & Nachfeld \\
\hline (2) Die Ehefrau & findet & $\begin{array}{l}\text { tatsächlich schnell } \\
\text { das Portemonnaie, } \\
\text { das ein Geschenk } \\
\text { ihres Mannes ist. }\end{array}$ & & \\
\hline (3) & Findet & $\begin{array}{l}\text { die Ehefrau } \\
\text { tatsächlich schnell } \\
\text { das Portemonnaie, } \\
\text { das ein Geschenk } \\
\text { ihres Mannes ist? }\end{array}$ & & \\
\hline (4) & weil & $\begin{array}{l}\text { die Ehefrau } \\
\text { tatsächlich schnell } \\
\text { das Portemonnaie, } \\
\text { das ein Geschenk } \\
\text { ihres Mannes ist }\end{array}$ & $\begin{array}{l}\text { findet / } \\
\text { gefunden } \\
\text { hat / } \\
\text { gefunden } \\
\text { haben soll }\end{array}$ & \\
\hline
\end{tabular}

Während also die linke Satzklammer immer „da“ ist, fehlt der rechte Gegenpart bei einem einfachen Prädikatsverb. Linguisten behaupten aber, sie sei zwar nicht sichtbar, aber virtuell doch vorhanden. Wenn wir in (2) die Reihenfolge im Mittelfeld verändern - wir kommen auf diese Möglichkeit noch ausführlich zu sprechen - können wir den Relativsatz „hinten“ belassen, vgl. (2`). Wäre er auch dann noch Teil des Mittelfeldes, müsste auch die Besetzung der rechten Satzklammer unmittelbar nach dem Relativsatz möglich sein, also (1`). Dies jedoch ist ungrammatisch. Somit zeigt sich, dass der Relativsatz in (2') nach wie vor ausgeklammert ist, von unsichtbarer Hand.

(2`) Die Ehefrau findet das Portemonnaie tatsächlich schnell, das ein Geschenk ihres Mannes ist. 
(1`) *Die Ehefrau hat das Portemonnaie tatsächlich schnell, das ein Geschenk ihres Mannes ist, gefunden.

Der rechte Satzklammerteil ist auch sonst etwas eigenwillig: Sogar die abtrennbaren Vorderteile von Prädikatsverben, Verbpartikeln wie $a b$, aus, ein usw., müssen in Verberst- und Verbzweitsätzen in den „einfachen“ Tempora, also im Präsens und Präteritum, dorthin wandern: Legt die Ehefrau das Portemonnaie schnell in der Garderobe ab? Sie las ihre Bücher immer schnell aus. In den anderen, „zusammengesetzten“ Tempora und in Verbletztsätzen generell stehen diese Elemente dort, wo sie inhaltlich hingehören, im Wortverbund mit dem Verbstamm: Wird die Ehefrau das Portemonnaie schnell in der Garderobe ablegen? Sie hat ihre Bücher immer schnell ausgelesen. [...] weil sie ihre Bücher immer schnell ausliest / ausgelesen hat. Wie man sieht, können $a b$, aus usw. aber auch im Wortverbund noch durch das Perfektmorphem ge- vom Verbstamm getrennt sein. Oder auch durch das Infinitivmorphem zu wie in weil sie ihre Bücher immer schnell auszulesen hat. So instabil sind diese Verbindungen. Wir kommen darauf in Kapitel 7, Abschnitt 4.5 zurück.

In der rechten Klammer kommt bei den zusammengesetzten Tempora, zumal im Passiv, schon einiges zusammen: So kann dort eine längere Kette von Verbformen aneinandergereiht erscheinen, wie z. B.

(5) weil er es gefunden haben wird:

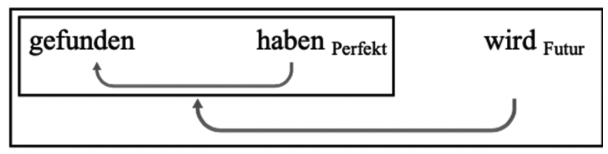

(6) weil es gefunden worden sein wird:

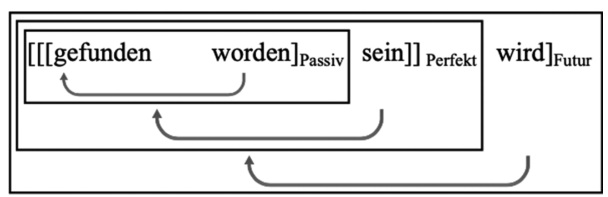

Abb. 14: Struktur von Verbketten in der rechten Klammer.

Hier wird, wie durch die Pfeile angedeutet, nach links „operiert“: Das übergeordnete Futurhilfsverb wird in (5) und (6) verlangt einen Infinitiv - und das darf auch wie hier ein Perfektinfinitiv sein. Der besteht in (5) aus dem Perfekthilfsverb haben und dem Partizip des Vollverbs finden. In (6) wird nun noch das Passiv eingeschaltet und zwar in Form des Infinitiv Perfekt Passiv. Dieser baut sich auf aus dem für das Passiv zuständigen Perfekthilfsverb sein, dem Partizip Perfekt des Passivhilfsverbs werden und dem Partizip des Vollverbs finden. 
Auch die Modalverben können, müssen, sollen oder wollen mischen hier mit, wie in diesen Variationen:

(7) weil er es gefunden haben soll

(8) weil es gefunden worden sein kann

(9) weil es nicht hat gefunden werden können

In (9) ist das höchste Glied der Verbkette, die finite Verbform hat, nach vorn „bewegt“ worden. Für diese Vorverlegung - die der Verarbeitung langer Ketten durchaus entgegenkommt - gibt es recht komplizierte Regeln, die aber nicht unbedingt befolgt werden, wie der Beleg (12) unten zeigt.

Damit nicht genug: Auch das Verb lassen oder die Wahrnehmungsverben sehen bzw. hören können sich noch einfügen und zwar als unmittelbare „Operatoren“ des Vollverbs, wie in den folgenden Belegen. Hier ist im Übrigen noch eine Besonderheit zu beobachten, der so genannte „Ersatzinfinitiv“. Im Perfekt heißt es standardsprachlich nicht, wie eigentlich zu erwarten, singen gehört hat, sondern eben (vgl. (12)) mit Infinitiv singen hören hat bzw. - noch besser hat singen hören:

(10) [...] weil sie den renommierten Schauspieler Jan Josef Liefers singen hören wollen (Rheinpfalz vom 11.03.2016)

(11) Klar darf einer nicht fünf Hochhäuser bauen, nur weil er nebenan eine Fabrikhalle hat stehen lassen (Tages-Anzeiger vom 18.01.2001)

(12) [...] weil sie mich schon so oft singen hören hat (Berliner Morgenpost vom 23.12.2004)

Im Englischen oder auch anderen Sprachen ohne Verbletztstellung kommt es nicht $\mathrm{zu}$ einer solchen Kettenbildung. Man vergleiche (13) mit dem englischen Gegenstück (14), wo - dies ist zumindest eine der möglichen Stellungen - die Adverbien certainly und thoroughly zwischen die einzelnen Teile der Verbgruppe eingefügt werden:

(13) weil er das Zimmer sicher gründlich durchsucht haben wird

(14) because he will certainly have thorougly searched the room 
Für Liebhaber der Felderstruktur sei noch darauf hingewiesen, dass jeder Teilsatz eines komplexen Satzes einerseits seine eigene Felderstruktur hat, andererseits aber auch in die Felderstruktur des Satzes, dessen Teil er ist, eingeordnet sein muss. Die Felderstruktur ist also ,rekursiv', wenn die Satzkonstruktion selbst rekursiv verläuft. Ich illustriere das hier am Beispiel von Die Ehefrau, der das Portemonnaie abhanden kam, hat einen verständnisvollen Mann. abhanden in abhanden kommen ist dabei übrigens auch als Verbpartikel einzuschätzen.

Tab. 7: Rekursion der Felderstruktur: ,IKl‘ steht für , linker Klammerteil, ,rKl‘ für ,rechter Klammerteil'.

\begin{tabular}{llll}
\hline Vorfeld & $\begin{array}{l}\text { linker } \\
\text { Klammer- }\end{array}$ & Mittelfeld & rechter Nach- \\
& teil & Klammer- feld \\
& teil & \\
\hline
\end{tabular}

(15)

$\begin{array}{lll}\text { Die Ehefrau, der das Portemonnaie abhanden kam, hat } & \text { einen } \\ \text { IKl } & \text { Mittelfeld } & \text { verständnis- } \\ \text { rKl } & \text { vollen Mann }\end{array}$

Die Belegung der Felder ist teilweise grammatisch geregelt, teilweise aber auch „frei“, das heißt, sie kann je nach den Intentionen der Sprecher oder der Schreiberin gestaltet werden. Diese raffinierte Mixtur aus Determination und Freiheit ist überhaupt kennzeichnend für die Topologie des Deutschen. So gilt für das Vorfeld zunächst einmal eine quantitative Beschränkung: Es darf, so heißt es, nur ein einziges Satzglied enthalten. Allerdings ist die Angabe ,ein Satzglied' in gewissen Fällen durchaus dehnbar. Während es in der Tat vollkommen „undeutsch“ ist, z. B. das Subjekt und das direkte Objekt zusammen ins Vorfeld zu setzen (wie etwa in ${ }^{\star} E r$ sie trifft morgen hier), kann man Umstandsangaben schon manchmal „zusammen“ nach vorn bringen. Bei dem Beleg „Gestern in Brüssel ist man dazu nicht in die Details gegangen“ (dpa vom 03.05.2013) stehen genau genommen zwei verschiedene Umstandsangaben im Vorfeld, eine temporale und eine lokale. Beide scheinen aber zu einer globaleren Situationsangabe zu verschmelzen. Und diese Möglichkeit entspricht auch unserem Bild von Ereignissen als durch die Kopplung von Raum und Zeit verankerte Szenarios.

Das Nachfeld hingegen ist quantitativ flexibler. Es beherbergt regelmäßig Nebensätze - etwa Relativsätze wie in den Beispielen oben - und Infinitivkonstruktionen. Deren Präferenz für dieses Stellungsfeld liegt auf der Hand: Versetzt man sie ins Mittelfeld, wird dort unter Umständen allzu viel an Information angehäuft. Und das, bevor der Adressat überhaupt weiß, was Sache ist. Denn das Vollverb steht oft immer noch aus. Aber auch Präpositionalphrasen, die sehr wohl im Mittelfeld ste- 
hen könnten, werden oft ausgeklammert. So kann es statt Sie hat das Portemonnaie schließlich an den richtigen Platz zurückgelegt auch heißen: Sie hat das Portemonnaie schließlich zurückgelegt an den richtigen Platz. Kasuskomplemente, also z. B. das Subjekt oder das direkte Objekt verweigern sich in aller Regel der Ausklammerung. Nur in dem hohen, nach Gesetzen des Rhythmus gestalteten Predigtton von Luthers Übersetzung der Worte Jesu kann es heißen: „Mir ist gegeben alle Gewalt im Himmel und auf Erden“ (Matthäus 28, 18). Hier befindet sich das Subjekt des Satzes im sein-Passiv hervorgehoben am Satzende, nach dem rechten Klammerteil. $^{16}$

Betrachten wir hingegen das direkte Objekt des letzten Beispielsatzes, so sagen wir nicht: ^Sie hat schließlich an den richtigen Platz zurückgelegt das Portemonnaie. Wenn uns gerade die Bezeichnung für das Objekt der Recherche nicht präsent ist, wenn wir noch einen Augenblick zum Nachdenken brauchen oder auch wenn wir die Sache besonders herausstellen wollen, dann müssen wir im Mittelfeld als „Platzhalter“ ein es einfügen. Erst dann dürfen wir das direkte Objekt hinter die rechte Klammer platzieren: Sie hat es schließlich an den richtigen Platz zurückgelegt, das Portemonnaie. Hier deutet dann das Komma - mündlich eine kleine Sprechpause - darauf hin, dass dieser Ausdruck noch weniger in den Satz „integriert“ ist als eine normale Nachfeldbesetzung. Linguisten sprechen dann von einem ,Nach-Nachfeld“ oder einem ,rechten Außenfeld‘. Besonders in mündlicher Rede nutzen wir Nachfeld und Nach-Nachfeld, wo es denn möglich ist. Das kommt der „allmählichen Verfertigung der Gedanken“ sehr entgegen. Mündlich und in informeller schriftlicher Kommunikation werden auch Ausdrücke ausgeklammert, die wir in wohlgeformten schriftlichen Texten nicht ausklammern würden, z. B. Zeitadverbien wie dann, jetzt, heute oder Ortsadverbien wie hier, dort oder auch andere Adverbien. So heißt es in einer elektronischen Kurznachricht: „Hey sister wann kommt ihr denn nach hamm heute?“17 (In diesem Fall ist es offen, ob heute das Nachfeld oder das Nach-Nachfeld besetzt.)

Das Nach-Nachfeld hat ein Pendant im Vor-Vorfeld bzw. linken Außenfeld. Die Besetzungsmöglichkeiten für beide Außenfelder ähneln sich. Wir können z. B. den Satz mit einer Anrede beginnen oder mit ihr ausklingen lassen: Statt „Frau Bundeskanzlerin, nehmen Sie die Wahl an?“ könnte es auch heißen: „Nehmen Sie die Wahl an, Frau Bundeskanzlerin?“ Im Vor-Vorfeld finden sich allerdings auch Elemente, die im Nach-Nachfeld nichts zu suchen haben. Nämlich Verknüpfungsausdrücke wie denn oder aber und allen voran natürlich und sowie oder. Auch die Besetzung der beiden Außenfelder sei für die gerade erwähnten Beispiele in einer Tabelle nachgezeichnet: 
Tab. 8: Erweiterung um Außenfelder.

\begin{tabular}{|c|c|c|c|c|c|c|}
\hline Vor-Vorfeld & Vorfeld & IKI & Mittelfeld & rKI & Nachfeld & Nach-Nachfeld \\
\hline (16) Hey sister, & wann & kommt & $\begin{array}{l}\text { ihr denn nach } \\
\text { Hamm }\end{array}$ & & heute? & \\
\hline $\begin{array}{l}\text { (17) Frau } \\
\text { Bundeskanzlerin }\end{array}$ & & Nehmen & Sie die Wahl & $a n ?$ & & \\
\hline (18) & & Nehmen & Sie die Wahl & $a n$ & & $\begin{array}{l}\text { Frau } \\
\text { Bundeskanzlerin? }\end{array}$ \\
\hline
\end{tabular}

Die Besetzung von Vorfeld und Mittelfeld birgt aber insgesamt die interessanteren Möglichkeiten: In diesen beiden Feldern schlägt sich vor allem nieder, wie die Intentionen des Sprechers, seine Gestaltungsabsichten und Schwerpunktsetzungen mit gegebenen grammatischen Restriktionen eine Verbindung eingehen. Wir wenden uns daher diesen beiden Feldern im Einzelnen zu.

\subsection{Was alles im Vorfeld stehen kann und was nicht dort stehen darf}

Im Vorfeld des deutschen Aussagesatzes befindet sich üblicherweise das Subjekt. Allerdings stehen, wie Auszählungen ergeben haben, nur etwas mehr als die Hälfte aller Subjekte im Vorfeld; ansonsten stehen Subjekte im Mittelfeld. Und umgekehrt: Nur ca. 57\% der Vorfeldbesetzungen in einem Korpus von Texten aus dem „Spiegel“ sind Satzsubjekte. ${ }^{18}$ Im Vorfeld tummeln sich alle möglichen anderen Satzglieder. Am zweithäufigsten sind adverbiale Bestimmungen, Supplemente, und zwar vor allem solche der lokal oder temporal situierenden Art. Aber auch Objekte jeglicher Couleur, Prädikativa und Anderes finden sich dort, wenn auch nur in geringerem Umfang.

Wieso steht mal das Subjekt vorn, mal aber auch z. B. das direkte Objekt? Bei einem Vergleich entsprechender Belege - es handelt sich jeweils um Vorkommen von der Mann (Subjekt) bzw. den Mann (direktes Objekt) im Vorfeld - muss der vorausgehende Kontext mit herangezogen werden: „Die Kirche feiert Paulus 2000. Geburtstag. Der Mann hat es sich nicht leicht gemacht“ (FOCUS vom 23.06.2008) versus „Deutschland, willst du wirklich mit dem Klopp durch die Wand? Den Mann hat schließlich nicht nur sein Witz und seine Eloquenz groß gemacht“ (Berliner Zeitung vom 05.12.2015). Von dem jeweiligen Mann hier der Apostel Paulus, dort der Erfolgstrainer Jürgen Klopp - ist bereits im Satz vorher und noch weiter zurück die Rede. Es geht um diesen Mann, der Mann ist Thema des Text(abschnitt)es. Lesen wir den Text laut vor, werden 
wir der Mann bzw. den Mann nicht besonders hervorheben. Wir werden den Ausdruck nicht durch Betonung gewichten. Denn es geht um das Bekannte, Erwartete, nicht um neue Information. Genau genommen betonen wir nur eine Silbe, in diesem Fall läge der Akzent auf der Silbe Mann. Aber die Wirkung des Akzents, die Hervorhebung, erstreckt sich auf die ganze Konstituente - sie könnte auch noch sehr viel komplexer sein wie etwa in den von mir erwähnten 'Mann.

Auch Passivierung kann in den Dienst der thematischen Kontinuität gestellt werden. Dies verdeutlichen die folgenden Variationen der Sequenz über den Apostel Paulus: Die Kirche feiert Paulus 2000. Geburtstag. Dem Mann wurde das Missionieren nicht leicht gemacht. / Der Mann wird als eigentlicher Gründer des Christentums betrachtet. Dabei ist das Vorfeld in der zweiten Variante durch das Passivsubjekt besetzt, in der ersten durch das Dativobjekt einer Passivkonstruktion.

Wir können daher als Hauptfunktion der Vorfeldbesetzung die Fortschreibung bekannter Information betrachten. Sofern es sich um Komplemente handelt, also in der Regel referenzielle Ausdrücke, ist im Vorfeld damit der Gegenstand genannt, um den es thematisch bereits gegangen ist und um den es nun auch in dem neuen Satz wieder geht. Dabei kann auch ein neuer Aspekt, eine neue Perspektive ins Spiel kommen, bei einer Person z. B. ihr Beruf, ihr Aussehen, ihre Familie usw. So könnte der zweite Satz des Paulus-Belegs z. B. lauten: „Sein Weg war nicht leicht.“ Nicht erstaunlich ist es daher, dass viele Vorfeldbesetzungen Personalpronomina der dritten Person sind, sind diese doch die thematischen Ausdrücke par excellence. Auch das Possessivum sein, wie in sein Weg, hat die Funktion der thematischen Fortführung, allerdings steht es nicht für ein Subjekt oder Objekt, sondern ein Genitivattribut, hier ist ja gemeint: der Weg des Mannes. Im modifizierten Thema ,der Weg des Mannes Paulus` wird das alte Thema auf der herabgestuften grammatischen Ebene als Attribut fortgeführt.

Zudem gibt es natürlich auch den Fall, dass zu einem neuen Thema übergegangen wird wie im folgenden Ausschnitt aus einer Reportage über einen Warnstreik: „Aber weitere Streiks im hessischen Handel werden folgen, kündigt Schmidt an. Eine Frau hat es sich auf einem Blumenkübel am Straßenrand gemütlich gemacht“ (Frankfurter Allgemeine vom 20.05.1999). Der neue Gesprächsgegenstand, hier ,eine Frau', wird typischerweise durch den indefiniten Artikel kenntlich gemacht, während definite Artikel, Demonstrativa oder eben Personalpronomina gebraucht werden, wenn Bekanntes fortgeführt oder elaboriert wird. In der „klassischen“ Version des linguistischen Thema-Konzepts hat man Bekanntheit und „Aboutness“ - ich finde keinen passenden deutschen Ausdruck - eng verknüpft und in erster Linie bekannte, fortgeführte Gesprächsgegenstände im Auge gehabt. Neuerdings hält man „Aboutness“, also die Frage, worüber der Satz handelt, und Bekanntheit auseinander. Den Gesprächsgegenstand nennt man nun eher auch topic oder eingedeutscht ,Topik‘. 
Nicht immer allerdings ist ein Ausdruck im Vorfeld unbetont. Schauen wir wieder zwei Belege an, jeweils mit Subjekt und direktem Objekt. Im „Spiegel“ vom 27. April 1998 heißt es: „Deutschlands führender Aufklärer, der Philosoph Immanuel Kant, kam nicht sehr weit. „Der Mann ist leicht zu erforschen', schrieb er, aber ,die Frau verrät ihr Geheimnis nicht‘.“ Im FOCUS vom 3. Februar 2018 liest man: „Es ist ein Geschenk und zeigt eine Frau mit Leggings und in der Mitte einen Stab, neben dem ein Mann steht. Den Mann hat Neo gemalt, die Frau hat Rosa gemalt.“ (Hier werden der bzw. den Mann - und selbstverständlich auch die Frau mit einem Akzent versehen, der den Kontrast verdeutlichen soll. Denn es geht jeweils um die Gegenüberstellung von Mann und Frau. (Dabei spielt es keine Rolle, dass im ersten Fall der Mann und die Frau „an sich“, als Gattungswesen, gemeint sind, im zweiten Fall dagegen spezifische „Personen“, nämlich Darstellungen in einem Bild des Maler-Ehepaars Neo Rauch und Rosa Ley.) Während unbetonte Ausdrücke den so genannten ,Hintergrund' der Information bilden, bilden betonte den Vordergrund oder ,Fokus‘. Das Thematische, das Bekannte oder auch Alte, bleibt in aller Regel im Hintergrund. Im Vordergrund steht natürlicherweise das Neue, das Noch-nicht-Dagewesene.

Und üblicherweise bildet die lineare Struktur das Verhältnis von Hintergrund und Vordergrund im Deutschen als ein Nacheinander ab. Der Informationsschwerpunkt liegt, in Form des Hauptakzents einer Äußerung, in der Regel „hinten“, meist am Ende des Mittelfelds. Wo er genau liegt, ist durch subtile Regeln bestimmt, auf die wir hier nicht eingehen. Man setze nur mal in Gedanken die Hauptakzente in den beiden Sätzen des Paulus-Belegs oben - ich markiere sie durch ' vor der akzenttragenden Silbe: Die Kirche feiert Paulus 2000. Ge'burtstag. Der Mann hat es sich nicht 'leicht gemacht.

Allerdings können wir den Hauptakzent auch anderswo setzen, wenn wir einen Informationsschwerpunkt setzen wollen, der nicht gleichläuft mit dem normalerweise linear gegebenen. Auch z. B. im Vorfeld, wie eben gesehen. Dabei kann dieses Hervorgehobene bereits bekannt sein wie in dem Beleg über das Bild des Ehepaars Rauch-Ley, oder auch nicht, wie in dem Beleg über Kant.

Kommen wir zurück zu dem großen Vorsprung des Subjekts vor dem Objekt als Vorfeldbesetzung. Zwar gibt es kaum deutsche Sätze ohne Subjekt und viele dieser Sätze haben kein Objekt. Doch wie die Untersuchung des Spiegel-Korpus zeigt, $^{19}$ beruht der Vorsprung nicht, jedenfalls nicht allein, darauf. Er könnte auch der besonderen Eignung des Subjekts zur Ausdruck von Topiks, also Gesprächsgegenständen, geschuldet sein. Diese wiederum könnte $u$. a. darauf beruhen, dass nur das Subjekt die Agens-Rolle kodieren kann, also handelnde Personen oder auch institutionelle Akteure, die normalerweise textsortenübergreifend auch die jeweils dort verhandelten „Agenda“ bestimmen und damit er- 
wartbarerweise auch präferierte Topiks sind. Und da das dann recht häufig für die aufeinanderfolgenden Sätze eines Textes gleichermaßen gilt, sind Subjekt-Topiks eben häufig auch thematisch im klassischen Sinne. Objekte machen dem Subjekt vor allem dann den Platz im Vorfeld streitig, wenn sie unmittelbarer an den Vortext anschließen als das Subjekt, wenn sie also Bekanntes kodieren, während das Subjekt Neues bringt. Das kann man ganz gut an dem Klopp-Beleg oben beobachten. Besonders schlagende Beispiele für Subjekte als Lieferanten neuer Information liegen übrigens vor, wenn uns Antworten auf Fragen wie „Was ist da / gestern / in China passiert?“ gegeben werden, etwa mit: „Da ist bedauerlicherweise ein Unfall passiert.“, oder „Gestern gab es im Süden Deutschlands einige heftige Gewitter.“ Oder „In China ist ein Sack Reis umgefallen.“ Will man ein solches neues, in den Fokus zu stellendes Subjekt - in der Regel ist es eine indefinite Nominalphrase - ans Mittelfeldende verfrachten und man hat keine Alternative für das Vorfeld, setzt man einfach ein Platzhalter-es dorthin: „ «Es haben sich hier schon einige Paare gefunden» sagt die Leiterin eines Single-Treffs“ (St. Galler Tagblatt vom 08.10.1998).

Aber genug der Spekulation. Tatsache ist, dass das Vorkommen von Objekten im Vorfeld, und zwar Objekten, die formal identisch sind mit dem Subjekt, Anlass zu Missverständnissen sein kann. Betrachten wir wieder einige Hohlspiegel-Belege: „Auch Einbrecher könnten Sensoren erkennen“ (Spiegel vom 01.03.2014). Zwar ist es im Sinne des Erfinders, dass Sensoren die Erkennungsleistung vollziehen, nicht etwa Einbrecher. Aber wir sind erst mal darauf geeicht, die Einbrecher als Subjekt und damit die Einbrecher als Akteure zu lesen. Was zwar nicht gewollt, aber gar nicht so abwegig ist. Eher schon abwegig ist das Missverständnis hingegen bei: „Zwei Gullydeckel haben Unbekannte von einer Brücke über der A 27 bei Walsrode im Heidekreis geworfen“ (Spiegel vom 10.09. 2016).

Der folgende Beleg ist besonders witzig, weil mit dem Fehlverständnis ,Satzteil vor dem Prädikat ist Subjekt‘ gleichzeitig das wörtliche Verständnis dieses Prädikats einhergeht, also die Verlobte wortwörtlich als rollend - z. B. den Berg hinunter, die Straße entlang - vorgestellt wird: „Das Verfahren bringt die Ex-Verlobte ins Rollen“ (Hohlspiegel, Spiegel vom 15.04.2013).

Im normalen Text betreffen diese Missverständnisse nur das direkte Objekt, weil Subjekt und direktes Objekt formal häufig zusammenfallen. In Schlagzeilen dagegen werden gerne die Artikel weggelassen, die z. B. Subjekt und Dativobjekt zuverlässig unterscheiden. So etwa in: „Polizei ging Diebespaar ins Netz“ (Hohlspiegel, Spiegel vom 30.03.2013). Gemeint ist: Der Polizei ging ein Diebespaar ins Netz. Wir tippen aber zunächst auf: Die Polizei ging einem Diebespaar ins Netz.

Solche Missverständnisse werden auch dadurch befördert, dass die ins Vorfeld versetzten Objekt-Satzglieder als Vordergrundelemente, als im Fokus stehend und 
somit betont zu lesen sind. Aber auch dies entspricht nicht dem gängigsten Muster, wie wir oben gesehen haben.

Ein Wort zu den adverbialen Bestimmungen als zweithäufigste Vorfeldbesetzung: Sie sorgen für den Zusammenhalt, die Kohärenz des Textes. Ihre Text-Funktion ist damit gar nicht so verschieden von der Funktion thematischer Subjekte oder Objekte. Allerdings liefern sie nicht Gegenstände, Topiks, sondern sie setzen den Rahmen für das Auszuführende, sei es durch (nähere) Situationsbestimmung, sei es durch argumentative Anbindung. Nehmen wir zwei Belege. Im ersten (aus der „Rheinpfalz“ vom 22.08.2014) berichtet ein Redakteur über seine berufliche Laufbahn, die er einem Mentor verdankt. Das ist die Person, auf die hier mit er Bezug genommen wird: „Er ging dann nach Ostdeutschland, da wollte ich nicht mit. Die können kein Pfälzisch. Dann hat mir der SWR ein Angebot gemacht, und dort bin ich heute noch.“ Die lokalen Ausdrücke da und dort und das temporale dann sind hier Vorfeldbesetzungen Neben dort ist im letzten Satz auch eine weitere - temporale - Angabe, nämlich heute vorhanden. Da es thematisch um Ortswechsel und „heimatliche“ Präferenzen geht, bietet sich das lokale dort eher als Vorfeldbesetzung an als das temporale heute. Im zweiten Beleg findet sich mit dagegen ein Ausdruck im Vorfeld, der einen Widerspruch zwischen aufeinanderfolgenden Aussagen anzeigt und uns damit weiter durch die Argumentation navigiert. Die vorausgehenden beiden Sätze haben das Subjekt im Vorfeld: „Coats folgt auf James Clapper. Er gilt als moderater Konservativer. Dagegen hat Trump mit Mike Pompeo einen republikanischen Hardliner zum Chef der CIA gemacht“ (Spiegel-Online vom 05.01.2017). Wir werden auf die Frage der Kohärenz im Text in Kapitel 6 zurückkommen.

Es sei nun noch der Blick gelenkt auf die - eher seltenen - aber für das Deutsche charakteristischen - Vorfeldbesetzungen mit einem infiniten Teil des Satzprädikats, also einem Partizip Perfekt oder einem Infinitiv oder gar einer Verknüpfung von beiden. In den folgenden Belegen steht das Partizip gefunden vorn, es ist, wie man so sagt, ,topikalisiert'. Im ersten würden wir dieses Wort wohl kaum besonders betonen, im zweiten schon eher: „Während des Zweiten Weltkrieges wurde in der Gemeinde Entlebuch Torf abgebaut, Ende der siebziger Jahre gar nach Erdöl gesucht. Gefunden wurde Erdgas, das ein paar Jahre gefördert wurde“ (Neue Zürcher Zeitung vom 31.12.2003). Der zweite Beleg thematisiert „eine groß angelegte Suche nach Rauschgift“. Dann heißt es: „Auch Drogenhunde wurden dabei eingesetzt. Gefunden wurde nichts“ (Nürnberger Zeitung vom 25.06.2013). Wie man sieht, geht es jeweils um das Minidrama ,gesucht - gefunden` . In den meisten Fällen ist bei dieser Art der Vorfeldbesetzung in ähnlicher Weise eine Erwartungshaltung bezüglich des Prädikatsinhalts bereits aufgebaut, der dann mit dem topikalisierten Prädikatsteil entsprochen wird. Um es noch ein bisschen komplizierter zu machen: Es könnte auch z. B. heißen: „[...] Erdgas gefunden 
wurde erst einige Jahre später.“ Oder gar: „[...] Erdgas gefunden haben werden sie nicht.“ Auch bei dieser Art der Vorfeldbesetzung erkennen wir unschwer eine typische Vorfeldfunktion wieder: die der Anknüpfung an das Bekannte oder Erwartete.

Wenn denn im Vorfeld so vieles möglich ist, welche Elemente können dort nicht erscheinen? Zeigen wir es an entsprechenden Beispielen:

Er hat mich ja nicht gesehen versus *Nicht hat er mich gesehen und ${ }^{\star} J a$ hat er mich nicht gesehen.

Ich hab es gewusst versus ${ }^{\star}$ Es hab ich gewusst.

Ich hab mich geschämt versus *Mich hab ich geschämt.

Da kann einem schlecht werden versus *Einem kann da schlecht werden.

Die Negationspartikel nicht ist also eines dieser Elemente, die Abtönungspartikel ja (und andere Abtönungspartikeln), das Pronomen es, sofern es nicht Subjekt ist oder Platzhalter für das Subjekt (wie z. B. in Es ist ein Stein ins Rollen gekommen), ein reflexiv zu verstehendes Pronomen, das fest zum Verb gehört wie bei sich schämen, sich brüsten und das generische einem (bzw. einen), das die fehlenden Formen von man ersetzt.

Ist das nur ein zufälliges Sammelsurium, oder folgt das Verbot aus den Eigenschaften oder Funktionen des Vorfelds? So ganz klar ist mir das nicht. Immerhin so viel: mich/dich/sich/uns/euch bei schämen ist nicht betonbar, während die Reflexiva bei anderen Verben durchaus betonbar sind - weil sie eben nicht alternativlos sind - und dann auch im Vorfeld stehen können: „Sich hat sie aufgegeben, die Kinder nicht“ (Berliner Zeitung vom 10.08.2009). Abtönungspartikeln wie ja, halt, eben, ruhig tragen keinen Akzent. Auch einen/einem ist in diesem Sinne nicht betonbar. Versehen wir es mit Akzent, haben wir wieder die Alternativen vor Augen, einen und andere. Dann kann der Ausdruck im Vorfeld stehen: „Er habe nicht mehr viele Freunde, heißt es. Einen hat er noch“ (Die Presse vom 20.08.2003). Auch es ist nicht betonbar - allerdings gilt das generell, also auch für ein Subjekt-es.

nicht schließlich ist durchaus betonbar: „Nein ich habe das 'nicht getan!“ kann man antworten, wenn hartnäckig nachgefragt und einem eine Tat unterstellt wurde. Allerdings ist das eher ein Sonderfall: Hier steht die Negation eines Sachverhalts selbst ganz im Fokus. Im „Normalfall“ negieren wir die Satzaussage; die Prädikation in einem weiteren oder engeren Sinne steht also im Fokus. Der Akzent liegt dann üblicherweise auf dem Vollverb, dem Prädikativ oder einem Satzglied, das eng zum Vollverb gehört, das als Teil der Prädikation zu verstehen ist: $E r$ hat mich nicht ge'sehen. Da braucht einem nicht 'schlecht zu werden. Er hat es sich nicht 'leicht gemacht. Würde man nicht ins Vorfeld versetzen, ginge der 
unmittelbare Kontakt zum eigentlichen Negationsbereich verloren. Auch andere ,Fokuspartikeln‘, z. B. ausgerechnet, lediglich oder sogar können nicht alleine das Vorfeld besetzen. Sie müssen das von ihnen Fokussierte mitnehmen: „Sogar einen Hund habe jemand mal auf sie losgelassen“ (Spiegel-Online vom 23.01.2018) klagt eine Betroffene. Entsprechend könnte es auch heißen „Nicht einen Hund habe jemand mal auf sie losgelassen, sondern [...]“.

So gesehen könnte man die Fälle nicht im Vorfeld zugelassener Elemente folgendermaßen zusammenfassen: Nicht hervorhebbare Konstituenten und solche, die von ihrer Hervorhebungsdomäne abgetrennt würden, sind im Vorfeld ausgeschlossen. Nicht erfasst wäre jedoch das Subjekt- und das Platzhalter-es.

\subsection{Was alles wo im Mittelfeld stehen kann}

Das Mittelfeld ist ein weites Feld: Es kann, wie gesagt, im Prinzip alle Satzteile außer dem Prädikat beherbergen. Wie die einzelnen Konstituenten aufeinander folgen, ist teils grammatisch geregelt, teils folgt es unseren Gewichtungswünschen.

Beginnen wir hier mit den Gewichtungswünschen. Dies hat uns ja gerade auch für das Vorfeld beschäftigt.

Man stelle sich also vor, eine Aussage liege uns - im Kopf oder auf einem Datenträger - in ihrer normalen Reihenfolge und mit ihrer „Normalgewichtung“ vor, z. B. so:

Ich habe meinem Freund gestern mein 'Fahrrad geliehen.

In der Normalreihenfolge und der Normalgewichtung sind, so kann man argumentieren, die linearen Folgen und Akzentverteilungen quasi kondensiert, die Sprecher und Schreiberinnen des Deutschen über Jahrhunderte hinweg praktiziert haben, wenn sie mit dieser und analog gestalteten Aussagen einfach nur pauschal den Inhalt transportieren wollten. Das heißt, wenn sie das aussagen wollten, was sich aus der Komposition der Teile ergibt, - ohne einzelne Konstituenten besonders hervorzuheben. Die Gestaltung sollte alle in ihr verborgenen Optionen offen halten.

Wenn die Normalgewichtung, was die drei Mittelfeld-Satzglieder meinem Freund, gestern und mein Fahrrad angeht, nicht unseren Wünschen entspricht, können wir einfach statt meinem Freund eines der beiden anderen Satzglieder betonen. Effektiver ist es aber, diese Aktion auch mit einer Änderung der Reihenfolge $\mathrm{zu}$ verknüpfen und das besonders gewichtige Satzglied in Richtung Mittelfeldende zu bewegen, wie in:

„Ich habe gestern mein Fahrrad meinem 'Freund geliehen.“ Hier wäre meinem Freund im Fokus. 
Oder:

„Ich habe meinem Freund mein Fahrrad 'gestern geliehen.“ Hier wäre gestern im Fokus.

Dies ist im Prinzip die Wirkungsweise der Gewichtsverteilung im Mittelfeld.

Wie steht es aber nun mit der Normalreihenfolge im Mittelfeld? Schön wäre es, wenn man da einfach ein einziges grammatisches Prinzip nennen könnte, z. B. ,Subjekt steht vor dem direkten Objekt, dieses vor dem indirekten Objekt bzw. dem Genitivobjekt, dieses wiederum vor einem Präpositionalobjekt‘. Diese Regel spiegelt die Hierarchie unter Subjekt und Objekten wider. So einfach ist es allerdings nicht. Wie auch in anderen grammatischen Bereichen überlagern sich mehrere Regeln oder vielmehr: Mehrere unterschiedlich gewichtige Regeln kommen hier ins Spiel. In der IDS-Grammatik werden insgesamt vier Regeln für die Ordnung der Komplemente im Mittelfeld genannt; die Supplemente bleiben erst einmal außen vor. Ich nenne diese Regeln hier kurz nach Maßgabe ihres Gewichts. Gewicht heißt hier übrigens, dass eine gewichtigere Regel vor den jeweils nachgeordneten anzuwenden ist bzw. umgekehrt betrachtet, dass weniger gewichtige Regeln durch die gewichtigeren überschrieben werden.

(Regel 1) Prototypische Komplemente zuerst

(Regel 2) Pronominale Komplemente zuerst

(Regel 3) Das Belebte zuerst

(Regel 4) Das in der Subjekt-Objekt-Hierarchie Übergeordnete zuerst

Zwar können wir diese Regeln nicht an einem einzigen Beispiel gleichzeitig zeigen. Aber mit Abwandlungen unseres Beispielsatzes oben wird es gehen. Der Satz Ich habe meinem Freund mein Fahrrad geliehen belegt Regel 3: Das Komplement, das etwas Belebtes bezeichnet, nämlich meinem Freund, steht vor dem, das etwas Unbelebtes bezeichnet, nämlich mein Fahrrad. Der Nebensatz weil ihm das diese Frau geliehen hat belegt Regel 2 und wiederum Regel 3: Die beiden Pronomina ihm und das stehen vor dem nicht-pronominalen Komplement diese Frau, und die Pronomina folgen dem Belebtheitsprinzip. Der Satz Ich habe meinem Freund das Fahrrad in die Garage gestellt belegt neben Regel 3 auch Regel 1: Die prototypischen Komplemente, nämlich das indirekte Objekt meinem Freund und das direkte Objekt das Fahrrad stehen vor dem nicht-prototypischen, nämlich dem Adverbialkomplement in die Garage. Um die am wenigsten gewichtige Regel zu demonstrieren, müssen wir Beispiele wählen, bei denen die höheren Regeln nicht zum Zuge kommen können, es also z. B. nur um prototypische Komplemente geht, die sich in Sachen Pronominalität und Belebtheit nicht unterscheiden: weil das Motorrad mein Fahrrad mit Dreck bespritzt hat. Hier steht das unbelebte Subjekt vor dem unbelebten direkten Objekt, das wiederum vor dem Präpositionalobjekt steht. Analog mit pronominaler Realisierung: weil etwas das damit bespritzt hat. 
Versuchen wir, den Sinn dieser Regeln zu ergründen. Allerdings führt auch hier die Sinnsuche eher ins Spekulative. Regel 1 ist vielleicht darin begründet, dass die weniger prototypischen Komplementarten, die Prädikativ- und Adverbialkomplemente, enger an das verbale Prädikat gebunden sind, das ja oft und möglicherweise sogar in der Grundreihenfolge, vgl. dazu das Ende dieses Abschnitts - in der rechten Klammer steht. Diese Komplemente wären dann ganz nah bei dem Ausdruck platziert, auf dem sie inhaltlich operieren. Die Regeln 2 und 3 kann man möglicherweise zurückführen auf die Eigenschaften der Thematizität oder Topikalität, die in entsprechenden Ausdrücken angelegt ist. Pronomina sind in aller Regel thematisch, beziehen sich auf Vorerwähntes oder Bekanntes. Wenn ein Pronomen im Vorfeld keinen Platz findet, z. B. weil Sprecherin oder Schreiber einer Umstandsangabe den Vorzug gegeben hat, wird es möglichst an den Mittelfeldanfang gestellt, also in den Hintergrundbereich der Äußerung. Sind auch andere Komplemente pronominal besetzt, wiederholt sich der Vorgang. So kommen ganze Bündel von Pronomina an dieser Stelle zusammen wie bei weil ich es ihm [...] / dass er sie dessen [...] / wo du sie mir [...] usw. Belebte Ausdrücke wiederum charakterisieren, wie oben skizziert, vorzugsweise Topiks und damit ggf. Akteure oder aber, insbesondere in Form des Dativobjekts, andere Mitspieler eines Szenarios, die ihrerseits häufig im vorangehenden Text oder Diskursverlauf schon erwähnt worden waren. Regel 4 schließlich liefert sozusagen das syntaktische Substrat der Linearisierungsoptionen im Mittelfeld. Wir müssen auch dann wohlgeordnet reden und schreiben, wenn keine der semantisch oder pragmatisch motivierten Regeln greift.

Es würde zu weit führen, auch auf die Stellung der Supplemente im Mittelfeld im Einzelnen einzugehen. Ich belasse es bei der Nennung eines allgemeinen Prinzips: Supplemente können generell an verschiedenen Positionen im Mittelfeld auftreten, vor den Komplementen (außer pronominal realisierten), zwischen und nach ihnen. Sind mehrere Umstandsangaben vorhanden, so ist bei normaler Gewichtung ihre Reihenfolge durch das Prinzip ,Was einen größeren Wirkungsbereich hat, kommt zuerst' bestimmt. Illustrieren wir das an einem konstruierten Beispiel mit möglichst vielen Umstandsangaben:

weil ihn der Dackel [angeblich] [gestern] [am Gartentor] [laut] angebellt hat

Das Supplement angeblich hat den weitesten Wirkungsbereich, nämlich die ganze Aussage, deren Gültigkeit durch angeblich abgeschwächt wird. gestern und am Gartentor bringen Spezifikationen des Geschehens, des ganzen Sachverhalts ins Spiel. Zwischen ihnen ist nicht unbedingt ein Bereichsunterschied auszumachen. Immerhin aber ist eine zeitliche Spezifikation mit sehr viel mehr parallel verlaufenden Ereignissen vereinbar als eine örtliche. In diesem Sinne hat erstere einen weiteren Wirkungsgrad. In der Regel geht eine temporale Bestimmung einer lokalen 
im deutschen Mittelfeld voraus. Der Ausdruck laut hat den engsten Wirkungsbereich, er bezieht sich nur auf das Bellen, also die Prädikation. Was übrigens die Abtönungspartikeln angeht, so können sie einzeln oder zu mehreren an verschiedenen Stellen im Mittelfeld - und nur dort - auftreten. Ich deute das durch an den entsprechenden Positionen in Klammer gesetztes ja an:

weil ihn der Dackel (ja) [angeblich] (ja) [gestern] (ja) [am Gartentor] (ja) [laut] angebellt hat An dieser Stelle bietet sich der Vergleich mit dem Englischen unmittelbar an. Dort herrscht, was die Reihenfolge von adverbialen Bestimmungen angeht, geradezu ein „Spiegelbild“ zum Deutschen. König/Gast ${ }^{20}$ zeigen dies an folgenden beiden übersetzungsäquivalenten Sätzen:

She has worked [on her boat] [with great care] [in the garden] [the whole time] [today]

Sie hat [heute] [die ganze Zeit] [im Garten] [mit großer Sorgfalt] [an ihrem Boot] gearbeitet

Die Autoren bringen diesen Unterschied in Zusammenhang mit der unterschiedlichen Verbposition in beiden Sprachen. Das Verb leite im Englischen die „Verbphrase“ ein, also den gesamten Satz mit Ausnahme des Subjekts, während es in der Grundstellung im Deutschen an deren Ende stehe. Sie betrachten also wie viele andere Forscher die Verbletztposition bzw. zumindest die Position des Vollverbs in der rechten Klammer als die Grundreihenfolge im Deutschen. In der Tat haben wir für die Reihenfolge der Komplemente bei Regel 1 ja bereits die Nähe zum Vollverb in der rechten Klammer geltend gemacht. Nun scheint auch die Positionierung der Supplemente ikonisch von der Nähe zum Prädikat, zum Gehalt des Verbs bestimmt zu sein - mit jeweils spiegelbildlichen Folgen für das Englische und das Deutsche. Möglicherweise lässt sich das zugrunde liegende Prinzip auch auf andere Sprachen übertragen. Auch im Französischen und im Polnischen dürfte ähnlich wie im Englischen verfahren werden.

\subsection{Die lineare Ordnung in den Sprachen der Welt und in europäischen Sprachen}

In den vorangehenden Abschnitten habe ich in einigem Detail und doch im Wesentlichen beschränkt nur auf die Stellung der Komplemente die Topologie des deutschen Satzes dargestellt. Wie kann dieses doch recht facettenreiche Bild auf das notwendigerweise grobe Raster einer für alle Sprachen gültigen Wortstellungstypologie projiziert werden? Und wie verhalten sich vor diesem Hintergrund andere europäische Sprachen, zumal unsere Kontrastsprachen?

Wortstellung, in erster Linie die Wortstellung der fundamentalen Satzteile Subjekt (S), (direktes) Objekt (O), Verb (V) ist seit den Arbeiten von Joseph Greenberg in 
den 1960er Jahren eines der Aushängeschilder der Sprachtypologie. Die in dieser Tradition stehenden Artikel im „World Atlas of Language Structures“ (WALS) zu dieser Thematik ${ }^{21}$ ergeben vereinfacht folgendes Bild: Insgesamt gibt es sieben Möglichkeiten der Reihenfolge von S, O und V. Sechs davon ergeben sich durch Permutation der drei beteiligten Größen, als siebte Möglichkeit ist der Fall zu nennen, bei dem eine Sprache keine dominante Wortfolge hat (,lacking a dominant word order"). Alle sieben sind in dem Sample von 1377 Sprachen der Welt belegt. Weit vorn liegen jeweils SOV und SVO mit um die 500 einschlägigen Sprachen, um die 200 weisen keine dominante Folge auf, immerhin noch ca. 100 Sprachen haben VSO, die restlichen drei Ordnungen sind weit abgeschlagen. Was die regionale Verteilung angeht, so ist Europa neben den Regionen Afrikas südlich der Sahara sowie in Ost- und Südasien von China bis Indonesien und dem Westpazifik derjenige Teil der Welt, wo SVO vorherrscht. Die keltischen Sprachen an den westlichen Rändern Europas allerdings haben VSO. Baskisch und Sorbisch werden als SOV eingeordnet.

Von den europäischen Sprachen, die wir hauptsächlich im Auge haben, werden jedoch entgegen dem europäischen Trend nur Englisch, Französisch und Polnisch als Sprachen mit SVO klassifiziert. Deutsch und Ungarisch hingegen zählen nach WALS zu den Sprachen, denen eine dominante Stellungsfolge abgeht. Dabei sind die Gründe in beiden Fällen durchaus unterschiedlich. Für das Deutsche, wie auch für das Niederländische, wird der syntaktisch motivierte Wechsel zwischen der Folge SVO im Hauptsatz (des Aussagesatztyps) und der SOV-Ordnung, also der Verbletztstellung, im Nebensatz geltend gemacht. Beide Folgen werden hier als gleichberechtigt gesehen. Im Ungarischen hingegen ist deshalb nicht von einer dominanten Wortstellung auszugehen, weil gar nicht die syntaktischen Funktionen Subjekt und Objekt entscheidend sind, sondern die pragmatischen Funktionen Topik und Fokus. Radikal vereinfacht steht nämlich ein Topik - es können gelegentlich auch mehrere sein - vorn im Satz. Unmittelbar vor dem Verb aber befindet sich die Fokus-Konstituente. Die kann aus allen möglichen Satzgliedern, oder gar Teilen davon, bestehen.

Illustrieren wir das an der Wiedergabe des Beispiels Ich habe mein Fahrrad meinem 'Freund geliehen im Ungarischen: mein Fahrrad gelte als Topik, meinem Freund ist Fokus, das pronominale Subjekt wird nicht als eigene Konstituente ausgedrückt, sondern ist in das Verb integriert. Es ergibt sich also diese Folge:

Tab. 9: Topik und Fokus im Ungarischen.

\begin{tabular}{lll}
\hline Topik & Fokus & Verbales Prädikat \\
\hline a biciklimet & barátomnak & kölcsönöztem \\
\hline mein Fahrrad & meinem Freund & habe-geliehen-ich \\
\hline
\end{tabular}


Die Leser könnten nun einwenden, dass ja auch im Deutschen nicht unbedingt das Subjekt vorn, im Vorfeld, stehen muss, sondern sehr wohl auch eine Topik- und unter Betonung sogar alternativ eine Fokus-Konstituente. Der wesentlicher erscheinende Unterschied ist jedoch die Beschränkung auf genau ein Satzglied vorn im Deutschen, während das Ungarische erstens überhaupt kein Vorfeld kennt und zweitens das Prädikat praktisch an jeder Stelle stehen kann, z. B. ganz vorn, wenn es selbst fokussiert ist und kein Topik vorhanden ist. ${ }^{22}$ Aus der übergreifenden Sicht des Typologen spielen aber auch die Frequenz oder die Unmarkiertheit der Stellungsmuster eine Rolle: Während im Deutschen das Subjekt und seltener ein anderes Topik vorn steht, steht im Ungarischen das Topik, das Subjekt oder ein anderes Satzglied sein kann, vorn. Während das Deutsche, so sagt man, wie andere europäische Sprachen eine subjektprominente Sprache ist, ist das Ungarische eine topikprominente. Nun wird zwischen einer frühen Nennung des Topiks und der Erstposition des Subjekts oft ein Zusammenhang hergestellt. So könne letzteres ein Grammatikalisierungseffekt aus ersterem sein. Dafür spreche, dass das Subjekt ein präferiertes Topik sei. Das sei besonders evident in vergleichsweise flexiblen Sprachen wie dem Deutschen, wo Subjekte noch eindeutiger mit Topikalität assoziiert sind als in Sprachen wie Englisch oder Französisch, wo (fast) jedes Subjekt, ohne Beachtung der Topikalität, vorn stehe. Allerdings ist es keineswegs der Fall, dass Topiks grundsätzlich, also quasi biologisch fundiert, in den Sprachen der Welt am Äußerungsbeginn genannt würden. Für viele Sprachen gilt eher das Gegenteil. ${ }^{23}$

Aber auch die SVO-Sprachen Englisch, Französisch und Polnisch sind nicht über einen Kamm zu scheren. Während Englisch und Französisch im Aussagesatz recht strikt die Regel ,Subjekt vor Prädikat' befolgen, wird im Polnischen bei Geschehens-Prädikationen ein indefinites Subjekt gern im Bereich des Satzendes, auch nach dem Prädikat, positioniert. In der deutsch-polnischen Grammatik von Engel et al. wird das deutsche Beispiel „Auf dem Weg nach Rom ist ihm viel Leid widerfahren“ wiedergegeben mit „W drodze do Rzymu spotkało go wiele cierpień“ (wörtlich: Auf Weg nach Rom ereilte ihn viel (des) Leids). ${ }^{24}$ Unschwer erkennen wir eine Parallele zu der oben vermerkten Tendenz, im Deutschen indefinite Subjekte, zumal bei Geschehens-Prädikationen, im Bereich des Mittelfeldendes zu positionieren. Auch Englisch und Französisch implementieren auf ihre eigene Art die Rechts-Tendenz des „logischen“ Subjekts bei Geschehens-Aussagen. Im Englischen wird dann an der Subjekt-Stelle ein there eingefügt, das manche gar selbst als Subjekt betrachten. Im Französischen wird das Pronomen il ,es ‘ als „formales“ Subjekt eingefügt. Man vergleiche: „There appeared a ship on the horizon“ ,Da/Es erschien ein Schiff am Horizont“ und „Il est arrivé un malheur“ ,Es ist ein Unglück geschehen‘. ${ }^{25}$ Eine weitere Parallele kann man in der Bündelung von Personalpronomina erkennen. Im Deutschen finden sie sich ja möglichst 
dicht nacheinander am Mittelfeldanfang, im Französischen und anderen romanischen Sprachen werden sie, oder vielmehr ihre unbetonten Varianten, unmittelbar vor dem finiten Verb eingeschoben. Damit wird im Französischen scheinbar die obligatorische SVO-Ordnung gestört. Allerdings gelten die kleinen Ausdrücke gar nicht als vollgültige Wörter, sondern sie bilden mit dem finiten Verb eine Einheit, man spricht dann von ,Proklitika‘, also vorn angelehnten Ausdrücken wie in: Je te le donnerai demain ,Ich gebe es dir morgen'. Auch im Polnischen stellt man die Personalpronomina, im Beispiel $c i$,dir‘ und to ,das' gern unmittelbar vor oder auch nach das Finitum wie in: Dam ci to jutro oder Jutro ci to dam.

Können wir nun ein generelleres Fazit zur Satztopologie im Deutschen und darüber hinaus ziehen? Zumindest Folgendes scheint mir klar: Das Deutsche unterscheidet sich nicht so sehr von Sprachen wie Englisch, Französisch oder Polnisch, was die Anordnung nach dem Gewicht der Teile, nach ihrem Mitteilungswert angeht. Wie in diesen Sprachen gehen im Deutschen Hintergrundinformationen dem Vordergrund in der Regel voraus. Andererseits kann auch sprachübergreifend der Anfang der Äußerung, mit Unterstützung durch den Akzent - oder anderer aufwendigerer Strategien -, der Hervorhebung dienen. Das Deutsche zeichnet sich vor allem durch zwei Besonderheiten aus: die Klammerstruktur und die damit erzeugte Aufteilung in topologische Felder und die nur statistische, nicht obligatorische Besetzung des „normalen“ Satzanfangs mit dem Subjekt. Diese syntaktischen Besonderheiten haben eine ganze Reihe von Folgeeffekten: Da im Deutschen auch z. B. Umstandsangaben den „normalen“ Satzanfang, sprich das Vorfeld, bilden können, besteht keine Notwendigkeit, diese in eine Extraposition links außen zu versetzen, wie es im Englischen und Französischen oft der Fall ist. Man vergleiche: [Heute] trafen sich [die Außenminster] in Brüssel versus [Today] [the foreign ministers] met in Brussels versus [Aujourd'hui] [les ministres des Affaires étrangères] se sont assemblés à Bruxelles.

Was aufwendigere Hervorhebungsstrategien wie ,Spalt'- und ,Sperrsätze‘ angeht, so sind diese im Deutschen, etwa im Vergleich zu Englisch und Französisch seltener. Beispiel für einen französischen Spaltsatz ist „C'est mille francs que cela coûte“, Beispiel für einen englischen Sperrsatz ist „What I need now is a long cool drink“. ${ }^{26}$ Im Deutschen können wir ohne Weiteres entsprechend dem Englischen sagen: „Was ich jetzt brauche, ist ein großes kühles Getränk.“ Bei dem französischen Beispiel wird es schon schwieriger: ?? „Es sind tausend Francs, die das kostet.“ Man kann es aber auch einfach bei „normalen“ Sätzen und intonatorischer Hervorhebung belassen: „Ein großes kühles Ge'tränk brauche ich jetzt“ bzw. „'Tausend Francs kostet mich das“.

Abschließend vielleicht folgendes Fazit: Pragmatische Wortstellungstendenzen mögen von vielen Sprachen, z. B. in einer geografischen Region, geteilt werden. Es mag auch sprachübergreifende Grammatikalisierungstendenzen, wie die 
Herausbildung der Subjekt-Erststellung aus der Topikprominenz heraus geben. Einzelne Sprachen prägen aber solch übergreifenden pragmatischen Tendenzen durch syntaktische Beschränkungen ihren Stempel auf.

\section{Satz oder Nichtsatz: Das ist hier die Frage}

Im „Blog der Republik“ liest man zum 12. Februar 2017, dem Tag der Bundesversammlung, Folgendes: ${ }^{27}$

Es war bezeichnend, dass der frisch gewählte künftige Bundespräsident, der Sozialdemokrat Frank Walter Steinmeier, einen Satz des ersten Sozialdemokraten im Amt des deutschen Staatsoberhauptes an den Anfang seiner Rede in der Bundesversammlung stellte. Gustav Heinemann hatte 1969 gesagt: „Es gibt schwierige Vaterländer, eines davon heißt Deutschland!“

Satz wird hier verstanden im Sinne von ,in sich abgeschlossener Redebeitrag‘ oder auch ,vollständige Mitteilungseinheit‘. Wobei natürlich die Merkmale ,abgeschlossen' bzw. ,vollständig' immer als relativ zu verstehen sind, denn in Wahrheit sind ja alle Redebeiträge, die wir so machen, auf dem Hintergrund von bereits Gesagtem zu verstehen, als Teile unendlicher Diskurs-Geschichten. Aber mit einem abgeschlossenen Redebeitrag wie dem oben genannten können Hörer etwas anfangen; sie verstehen ihn und ordnen ihn in ihr Wissen ein. Oft handelt es sich auch um besonders markante Redebeiträge, die es nach Meinung derer, die sie zitieren, verdienen, als Leit- oder Merksätze, als Sentenzen (von lat. sententia ,Satz') aufbewahrt zu werden.

Bei diesem Verständnis von Satz kommt es nicht oder kaum auf die Form an. Dem steht das grammatische Verständnis des Ausdrucks Satz gegenüber, auf das wir uns bisher in diesem Buch und vor allem in diesem Kapitel bezogen haben. Machen wir dieses grammatische Verständnis von Satz hier noch einmal explizit: Ein Satz besteht aus einem finiten Verb und mindestens den vom Verb geforderten Komplementen. Dies ist eine syntaktische, eine formbezogene Definition. Sie hebt aber keineswegs auf eine willkürliche Form ab, sondern auf diejenige, die in den uns vertrauten Sprachen typischerweise die grundlegende semantische Einheit, die Proposition, also die Kombination von Prädikat und zugehörigen Argumenten ausdrückt; vgl. dazu Kapitel 2, Unterkapitel 6.

In dem Satz, den Gustav Heinemann und nach ihm Frank Walter Steinmeier äußerten, gibt es aber zwei finite Verben mit ihren jeweiligen Komplementen. Dieser Satz des Typs ,Redebeitrag‘ besteht also aus zwei grammatischen Sätzen. Offensichtlich steht es oft im Ermessen der Sprecherinnen oder der Schreiber, mehr als einen grammatischen Satz zu einem Redebeitrag zu erklären. In der 
Schrift setzen sie dann zwischen den einzelnen nur ein Komma oder einen Strichpunkt und erst am Ende einen Punkt, ein Frage- oder ein Ausrufezeichen. Mündlich senken oder heben sie nach dem ersten grammatischen Satz nicht etwa ihre Stimme, sie machen keine längere Pause, sondern fahren einfach auf etwa der gleichen Tonhöhe fort. Warum tun sie das wohl? Offensichtlich werden die Teile so enger aneinandergebunden. In unserem Beispiel etwa wird die durch den ersten Teil beim Hörer erzeugte Frage - welche Vaterländer mögen das wohl sein? - umgehend durch den zweiten Teil beantwortet, jedenfalls für diesen Kontext zureichend beantwortet. Allgemeiner könnte man sagen, dass Redebeiträge, die aus mehreren ,gleichgeordneten` Sätzen bestehen, wie in diesem Fall, unter eine einheitliche illokutive Kraft gestellt werden: Es werden nicht getrennte Behauptungen aufgestellt, sondern eine Behauptung, die sich in Teilbehauptungen gliedert. Das ist nach den Gesetzen der Logik und Mathematik nicht unbedingt ganz sauber, aber gelegentlich wirkungsvoll.

Auch die erste Mitteilungseinheit des obigen Zitats besteht aus zwei grammatischen Sätzen. Hier sind die Teile jedoch nicht gleichgeordnet, sondern wir haben es mit Unterordnung zu tun: Der dass-Satz ist als Nebensatz untergeordnet. Unterordnung heißt jedoch nicht notwendigerweise Verzichtbarkeit. In diesem Fall ist der Nebensatz Subjekt des ,Ganzsatzes‘, so könnte man die Kette von einem Punkt zum anderen nennen. Und als Subjekt natürlich unverzichtbar; das es am Satzanfang ist ja nur sein Platzhalter und hält ihm, dem eigentlichen Subjekt, die angestammte Stelle sozusagen warm. Der Nebensatz ist also im Ganzsatz untergeordnet, nicht etwa unter einen anderen Satz geordnet. Was übrig bleibt, wenn der dass-Satz entfällt, ist nämlich kein Satz, sondern nur der unvollständige Rest eines Satzes. So verhält es sich auch bei Objektsätzen oder allgemeiner gesagt bei allen Komplementsätzen, etwa dem $o b$-Satz in folgendem Beleg aus dem Märchen „Die Gänsehirtin am Brunnen“: „Der Graf wußte nicht, ob er weinen oder lachen sollte.“

Bei Supplementsätzen dagegen, z. B. weil- oder wenn-Sätzen, ist die traditionelle Sehweise, dass ein Nebensatz einem ,Hauptsatz' untergeordnet ist, eher angebracht: Der Nebensatz kann weggelassen werden und, was bleibt, ist immer noch ein Satz. Man prüfe es nach etwa an der traurigen Aussage der Schwester aus dem Märchen „Die zwölf Brüder“: „Ich will gerne sterben, wenn ich damit meine zwölf Brüder erlösen kann.“

Bei Satzgefügen mit untergeordneten Sätzen stellt sich die Frage ,ein Sprechakt oder mehrere?` nicht. Nebensätze haben keine eigene illokutive Kraft; nur der Ganzsatz wird als Behauptung, als Versprechen oder Aufforderung usw. verstanden. Und nur aus den Eigenschaften des Satzes, in dem oder dem der Nebensatz untergeordnet ist, lässt sich der Satzmodus als syntaktische Grundlage des Sprech- 
aktpotenzials erkennen, vor allem an seiner Verbstellung. Die Nebensätze sind ja ohnehin in aller Regel Verbletztsätze.

Im Deutschen müssen wir mit der Zwei- oder Mehrdeutigkeit von Satz leben. In der alltäglichen Kommunikation ist das nicht weiter problematisch. Auch andere Begriffe haben einen Interpretationsspielraum, der sich im Zusammenhang nicht störend bemerkbar macht und meist sogar auflöst. Auch das Wort Wort hat ja eine engere, „linguistische“ Bedeutung und eine weitere. Hier allerdings unterscheiden sich die Pluralformen: Mein Text hier enthält eine ganze Menge deutsche Wörter. Die Worte des Vorsitzenden Mao Tsetung, die in dem „kleinen roten Buch“, der „Mao-Bibel“, gesammelt wurden, waren Zitate aus dessen Reden, die immer aus mehr als einem Wort und oft auch aus mehr als einem Satz (in beiderlei Sinne) bestanden.

Die Sprachwissenschaft allerdings tut sich schwer mit der Doppeldeutigkeit von Satz. In der englischen und französischen Tradition unterscheidet man terminologisch zwischen sentence bzw. phrase und clause bzw. proposition, jeweils für die Mitteilungseinheit und die grammatische Einheit. In der Sprachwissenschaft des Deutschen hat sich eine solche Unterscheidung nicht eingebürgert. Eher arbeitet man mit näheren Bestimmungen von Satz in Komposita wie dem bereits erwähnten Ganz- oder auch Gesamtsatz bzw. Vollsatz ${ }^{28}$, Nebensatz usw.

Die enge Beziehung zwischen (grammatischem) Satz und Mitteilungseinheit, die zu der Doppeldeutigkeit geführt hat, gilt jedoch nur im Sinn von ,Alle Ganzsätze sind Mitteilungseinheiten', nicht in der Umkehrung. Nicht alle Mitteilungseinheiten haben nämlich die Form von Sätzen. Wir wissen alle, was gemeint ist, wenn in einer brenzligen Situation einer ruft: „Nichts wie weg hier!“ Das ist in der Situation sparsamer und effektiver als etwa der Satz „Laufen wir weg von hier!“ Solche Kurzformen, neuerdings als „dichte Konstruktionen“ bezeichnet, ${ }^{29}$ enthalten kein finites Verb; sie sind aber, was ihren propositionalen Gehalt und ihr illokutives Potenzial angeht, hinreichend vollständig: Die in der Situation Anwesenden werden aufgefordert, sich von ihrem Aufenthaltsort wegzubegeben manchmal richtet der Sprecher sich damit auch nur an sich selbst. Diese Kurzform scheint zwar fest gefügt zu sein - in DeReKo ist sie wortwörtlich $179 \mathrm{Mal}$ belegt. In Wahrheit ist sie aber doch recht flexibel. Man findet unter Anderem: „Nichts wie hin / los / raus / heim!“ Auch Formen wie „Nichts wie rein in die gute Stube / raus aus dem Bett / heim an die Wärme!“ sind belegt. Dabei wird zur Richtung, in die es gehen soll, noch der Zielort näher spezifiziert. Im Grunde dient ja das formelhafte „nichts wie“ nur zur Verstärkung. Es kann also auch weggelassen werden wie in „Weg hier!“, „Ab nach Kassel!“, „Raus in die frische Luft!“. Daneben kann auch das, was sich wegbewegen oder wegbewegt werden soll, explizit genannt werden. In der 2. Szene des 2. Aktes von Schillers „Kabale und Liebe“ empört sich Lady Milford angesichts der Juwelen, eines Geschenks 
des Herzogs, die dieser mit dem Verkauf von Landeskindern als Soldaten nach Amerika erworben hat: „Weg mit diesen Steinen - sie blitzen Höllenflammen in mein Herz!“ Die mit-Phrase - vgl. auch kurz: „Weg damit!“ - hat hier keine ihrer üblichen Lesarten. Weder nennt sie das Instrument noch eine Begleitperson oder einen Begleitumstand. Es scheint sich zudem um eine Verwendungsweise von mit zu handeln, die spezifisch für das Deutsche ist.

Handlungsanweisungen scheinen eine besondere Affinität zu solchen Verdichtungen zu haben. Das nimmt nicht Wunder, kommt es doch gerade in solchen Situationen oft auf prägnante Anweisung und prompte Ausführung an. Bei der zuvor geschilderten Form blieb die genaue Art der Aktion offen; immer ging es um Bewegung oder Transfer. Geht es aber um eine konkrete Handlung beliebiger Natur, wird ein verbaler Ausdruck genutzt, der aber in infiniter Form vorliegen kann wie in: „Hier geblieben!“, „Die Hände hochnehmen!“, „Nicht schießen!“, „Sofort alles dichtmachen!“

Andere Sprechakttypen, Aussagen oder Fragen etwa, werden in der Regel nur in speziellen Gattungen, wie der Schlagzeile oder allgemeiner im Telegrammstil, in einer Form ohne finites Verb formuliert. So findet sich auf der ersten Seite der aktuellen Ausgabe meiner Tageszeitung eine Schlagzeile mit dem Partizip Perfekt eines Verbs, nämlich „Armbrustfall aufgeklärt“, eine mit einem Adjektiv als Prädikatsausdruck, nämlich „FDP-Chef Theurer offen für Grün-Gelb“ und eine, in der eine Frage formuliert ist: „Neuer Standort fürs Nahostarchiv?“ ${ }^{30}$ In den beiden ersten Fällen ist neben dem Prädikatsausdruck (aufgeklärt bzw. offen für Grün-Gelb) auch das jeweilige Argument artikuliert (Armbrustfall bzw. FDP-Chef Theurer). Und damit ist die jeweilige Proposition semantisch auch abgeschlossen. Der letzte Fall ist etwas komplexer. Es wird zwar nur eine Nominalphrase genannt und mit Fragezeichen versehen. Aber offensichtlich, so schließen wir, wird auf diese Weise nach einer Bereitstellung des von der Nominalphrase Bezeichneten, also der künftigen Existenz eines neuen Standorts fürs Nahostarchiv gefragt. Man kann die Kurzform aber nicht einfach als Weglassen von „Gibt es“ erklären, denn dann müsste ja die Nominalphrase im Akkusativ, nicht, wie hier, im Nominativ stehen. Dichte Konstruktionen sind also keine Ellipsen, Weglassungen von sprachlichem Material. Diese gibt es schon auch, und wir gehen auf diesen Typ im nächsten Kapitel näher ein. Vielmehr handelt es sich um satzunabhängige Konstruktionsformen, die eine weniger transparente Struktur als Sätze und einen eingeschränkteren Anwendungsbereich haben. Gut platziert, können sie dort aber besonders ökonomisch und effektiv wirken.

Sätze und Kurzformen bzw. dichte Konstruktionen kommen also unterschiedlichen Interessen entgegen, obwohl sie beide einen propositionalen Gehalt und eine Illokution ausdrücken und damit als kommunikative Einheiten fungieren können: In Sätzen wird z. B. notwendigerweise durch Tempus und Modus des finiten Verbs 
die zeitliche Verortung und der Realitätsbezug der Information kodiert. Zudem liegen die syntaktischen Funktionen der Teile vergleichsweise offen. Sätze sind also prädestiniert für den Austausch präziser Information auf der Darstellungsebene im Sinne Bühlers. ${ }^{31}$ Wo es hingegen um Kürze, aber auch nach Bühler um Ausdruck und Appell geht, können Kurzformen durchaus konkurrieren. 